Portland State University

PDXScholar

1981

\title{
A study of Leslie model under stochastic environments
}

Kamran Shaukat

Portland State University

Follow this and additional works at: https://pdxscholar.library.pdx.edu/open_access_etds

Part of the Population Biology Commons, and the Statistical, Nonlinear, and Soft Matter Physics Commons

Let us know how access to this document benefits you.

\section{Recommended Citation}

Shaukat, Kamran, "A study of Leslie model under stochastic environments" (1981). Dissertations and Theses. Paper 3112.

https://doi.org/10.15760/etd.3109

This Thesis is brought to you for free and open access. It has been accepted for inclusion in Dissertations and Theses by an authorized administrator of PDXScholar. Please contact us if we can make this document more accessible: pdxscholar@pdx.edu. 
AN ABSTRACT OF THE THESIS OF Kamran Shaukat for the Master of Science in Physics presented August 5, 1981.

Title: A Study of Leslie Model Under Stochastic Environments.

APPROVED BY MEMBERS OF THE THESIS COMMITTEE:

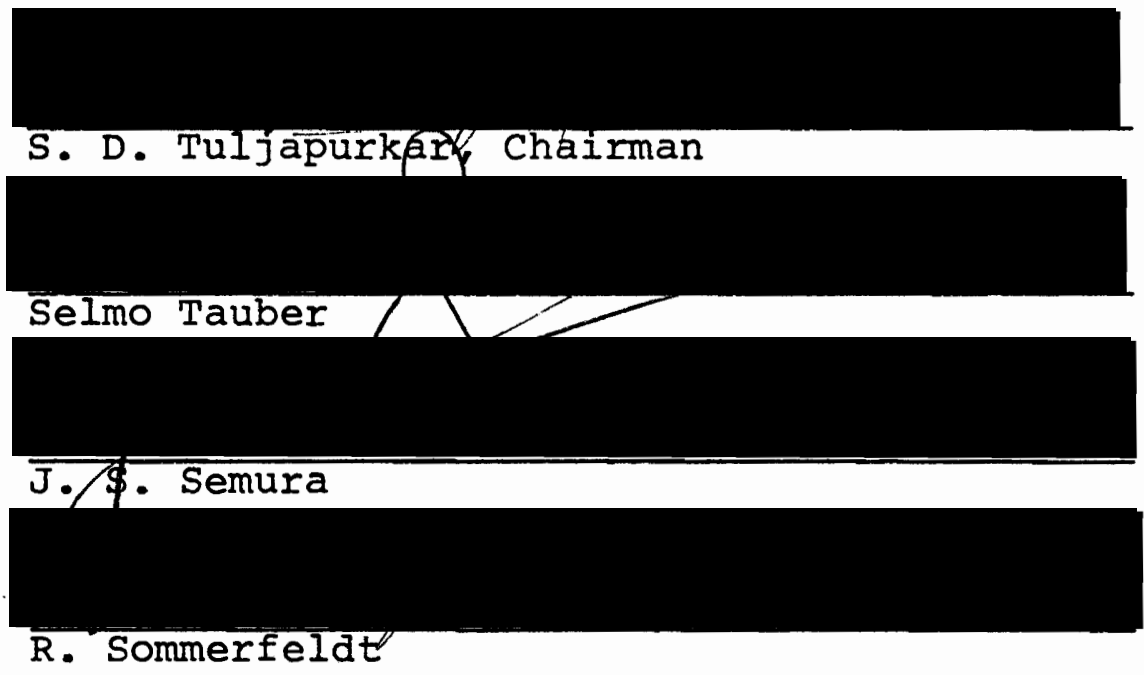

The prediction and analysis of changes in the numbers of biological populations rest on mathematical formulations of demographic events (births and deaths) classified by the age of individuals. The development of demographic theory when birth and death rates vary statistically over time is the central theme of this work. A study of the standard Leslie model for the demographic dynamics of populations in variable environments is made. At each time interval a Leslie matrix of survival rates and fertilities of a population is chosen according to a Markov process and the 
population numbers in different age classes are computed. Analytical bounds are developed for the logrithmic growth rate and the age-structure of a population after long times. For a two dimensional case, it is shown analytically that a uniform distribution results for the age-structure if the survival rate from the first to the second age-class is a uniformly distributed random quantity with no serial autocorrelation. Numerical studies are made which lead to similar conclusions when the survival rate obeys other distributions. It is found that the variance in the survival parameter is linearly related to the variance in the agestructure. An efficient alogrithm is developed for numerical simulations on a computer by considering a time sequence of births rather than whole populations. The alogrithm is then applied to an example in three dimensions to calculate a sequence of births when the survival rate from the first to the second age-class is a random parameter. Numerical values for the logrithmic growth rate and the logrithmic variance for a population and the probability of extinction are obtained and then compared to the analytical results reported here and elsewhere. 
A STUDY OF LESLIE MODEL

UNDER STOCHASTIC ENVIRONMENTS

by

KAMRAN SHAUKAT

A thesis submitted in partial fulfillment of the requirements for the degree of

MASTER OF SCIENCE

in

PHYSICS

Portland State University 
TO THE OFFICE OF GRADUATE STUDIES AND RESEARCH:

The members of the committee approve the thesis of Kamran Shaukat presented August 5, 1981.

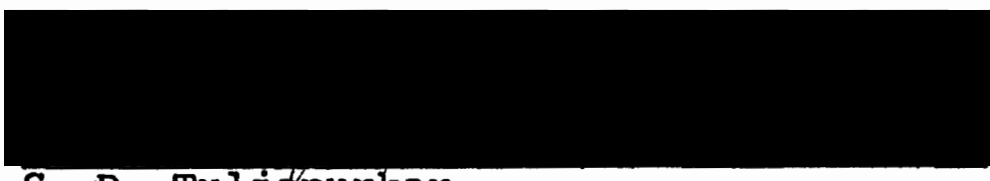

S. D. Tuljapurkar

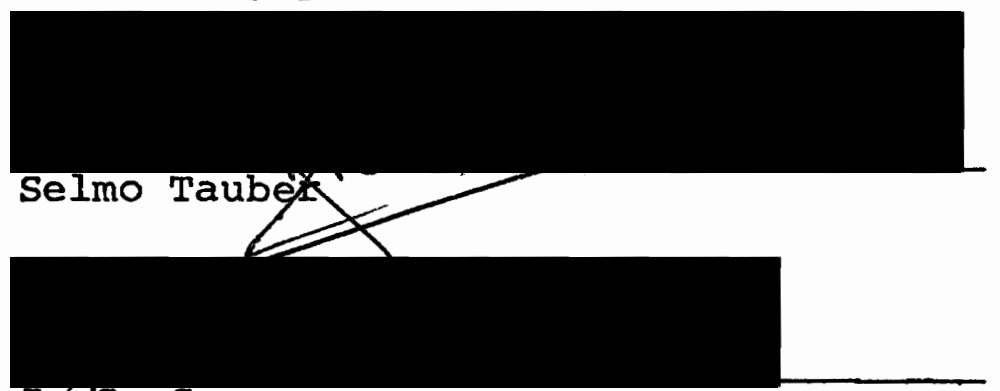

J/s. semura

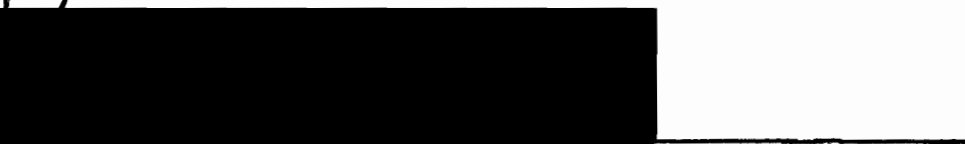

R. Sommerfeldt

APPROVED :

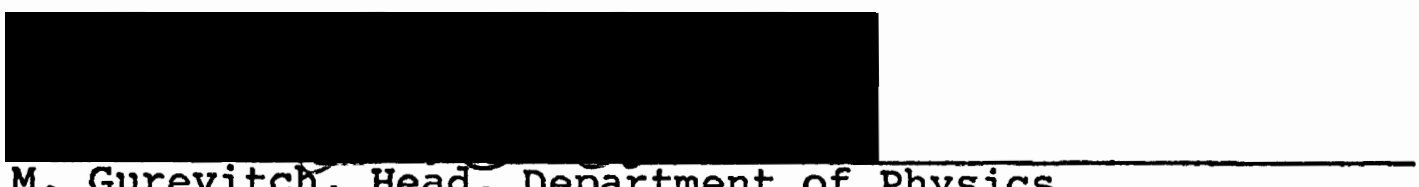

M. Gurevitch, Head, Department of Physics

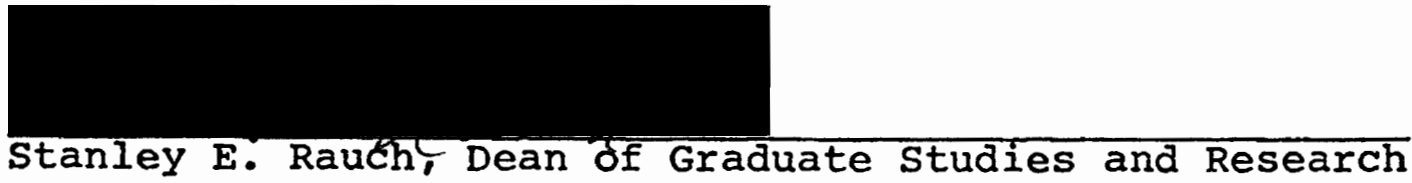




\section{ACKNOWLEDGEMENT}

I am grateful to Dr. Shiripad D. Tuljapurkar, my teacher and friend, for introducing me to this very interesting problem. I wish to express my great appreciation for his remarkable prowess with the complex ideas in physics and mathematics, his refreshing sense of humour, and above all for his help and guidance throughout this work.

I appreciate the encouragement and support given to me by Dr. Mark Gurevitch, Dr. Jack S. Semura, Ray Sommerfeldt, Mrs. Dawn Dressler and the remaining teachers and staff in the Physics Department. I wish to thank my friends Bill Brown, Jullian Hobbs, David.Lezak, and Scott Cohen for providing congenial atmosphere. I also thank Leslie Fitzpatrick for typing the manuscript.

This work in part was supported by a grant from the Portland State University Foundation. 
TABLE OF CONTENTS

PAGE

ACKNOWLEDGEMENTS

iii

LIST OF TABLES

LIST OF FIGURES

CHAPTER

I INTRODUCTION..$\cdot$. . . . . . . . .

II THE BACKGROUND ON POPULATION MODELS IN ECOLOGY

a) The Deterministic Models . . . . . 5

b) The Stochastic Models ....... 8

III LESLIE'S MODEL • . • . . . • • • • •

IV BOUNDS FOR POPULATION GROWTH RATE AND AGE STRUCTURE IN VARIABLE ENVIRONMENTS

V A TWO DIMENSIONAL EXAMPLE

Results

VI SIMULATION IN THREE DIMENSIONS . . . . .

Example . . . . . . . . . . 31

Results ............ 32

REFERENCES

APPENDIX A . . . . . . . . . . . . . . 


\section{LIST OF TABLES}

TABLE

PAGE

I Comparison of Numerical and Analytical Values of $a$ and $\sigma^{2}$.............. 


\section{IIST OF FIGURES}

FIGURE

PAGE

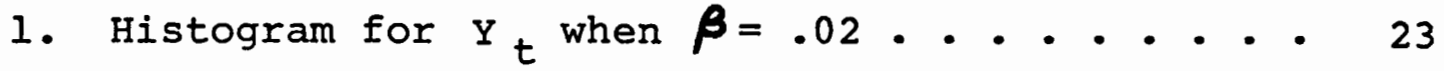

2. Histogram for $Y_{t}$ when $\beta=0.1 . . . . . . . ~ 24$

3. Histogram for $U_{t}$ when $\beta=0.1 . . . . . . .25$

4. Relation of Variance in the Random Parameter

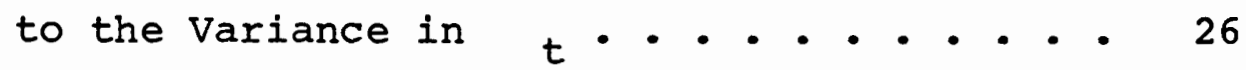

5. Graph Between the Logrithmic Growth Rate and the Logrithmic Variance . . . . . . 34

6. Plot of a vs. $\sigma^{2}$ when $E\left(p_{1}\right)$ is fixed but $m_{3}$ is allowed to have different values . . . . 35

7. Extinction Probability as a function of time . 37 


\section{CHAPTER I}

\section{INTRODUCTION}

The development of sound models for the time trajectory of population numbers, growth rate and extinction probabilities is of prime importance in ecological theory. Since such models are used to predict the environmental consequences of human activities, the strengths, weaknesses and domain of applicability of the models must be clearly understood to avoid costly ecological mistakes.

The foundation of most ecological models is the deterministic model of Malthus (1798) which describes the exponential growth of a single species population. The model was greatly developed in modern times by Lotka (1907), Sharpe and Lotka (1911) and Volterra (1926, 1937). Such models assume an unvarying exponential growth rate which is unreasonable in real communities, albeit, is common in laboratory populations unlimited by nutrients or space. Another shortcoming of Lotka-Volterra type models is that they consider populations as a whole disregarding their internal structure and its effects. For example, the birth and the death rates which are used to estimate the rate of natural increase depend largely on the ages (or sizes) of individuals in a population. Questions about the impact 
of infant mortality and the reproductive span, of great interest in human demography, cannot be addressed properly without looking into the age-structure of a population. More complex versions of the basic Lotka-Volterra type models employ continuous time and age variables to account for age-structure. An equivalent class of models employs matrix algebra to describe age-structure in discrete time intervals. Such models use a discrete time variable and a discrete age-scale and have been studied extensively under unvarying environments with age dependent birth and survival rates (Bernardelli, 1941; Lewis, 1942; Leslie, 1945, Keyfitz, 1968, Pielou, 1977). Use of these models permits us to predict the age or size composition of a population after long times along with the population number and the eventual rate of increase. The continuous time models will be referred to as Lotka models; the discrete time versions will be called Leslie models, following Lotka (1907) and Leslie (1945).

Further development in ecological theory depends on more realistic extensions of the deterministic models above to include temporal variability of environments. Changing environmental conditions induce fluctuations in the vital rates of a population so the results of deterministic models are no longer applicable to the dynamics of populations. Temporal fluctuations in the vital rates of a population can be predictable or random. Predictable variations 
at seasonal and other frequencies have been studied by several workers. It has been shown by Skellam (1967), MacArthur (1968), Coale (1972) and Gourley and Lawrence (1977) that periodicity in vital rates results in corresponding variations in population structure. For an arbitrary but known sequence of vital rates which applies over all future times, Lopez (1961) has proven the weak ergodic theorm of demography, i.e., after. long times any initial age distribution eventually converges to the same but not necessarily fixed age distribution and the same growth rate.

A more general approach to the problem would be to look at the Leslie model for age or size structured populations in temporally unpredictable environments. The Leslie matrix of vital rates is then randomly determined at each time interval. Following Pollard (1973) and Sykes (1969) the Leslie matrices for a particular realization are assumed to be independent random variables or are chosen according to a Markov chain. Several workers have studied the mean and variance of the population vector (Pollard, 1973; Sykes, 1969; Namkoong, 1972). Weissner (1971) and Athreya and Karlin (1971) have explored the question of asymptotic extinction of a population. Long run growth rates in unpredictable environments have been studied numerically by Boyce (1977) and analytically by Cohen $(1976,1977 a, b)$. Cohen (1977a) has also established the random version of the demographic weak ergodic theorm for Markov sequence of vital 
rates. Tuljapurkar and orzack (1980) have studied the analytical distribution of the asymptotic growth rate of populations, and a given numerical example of a two dimensional stochastic model.

Here further results are developed for variability in survival and mortality schedules under random fluctuations using Monte Carlo techniques and analytical methods. We have focused on the problem of age-structure and its distribution and have obtained various useful results. Next, an efficient alogrithm is developed for numerical work on the stochastic Leslie model. The alogrithm is applied in three dimensions to study the logrithmic growth rate and its variance, and examine the probability of extinction. The analytical approximations developed in Tuljapurkar (1981) are tested here against numerical calculations. 


\section{CHAPTER II}

THE BACKGROUND ON POPULATION MODELS IN ECOLOGY

\section{a) The Deterministic Models}

The central mathematical model of population due to Malthus (1798) describes a single species growing in an unvarying environment with fixed fertilities and mortalities; this model is the basis for deterministic models of multispecies ecological communities. The differential equation for the Malthusian model of change in population numbers with time is

$$
\frac{d x(t)}{d t}=r x(t)
$$

where $x(t)$ is population at time $t$, and $r$ is the net growth rate. The analogus difference equation when time is measured in discrete intervals is

$$
N(t+1)=m N(t),
$$

where $N(t)$ is the population number at time $t$ and $m$ is the net growth rate in discrete time. Note that for equivalence of these two models, $m=\exp (r)$.

In the above equations, population numbers change exponentially; a trend which cannot continue for long in actual populations which would either disappear or become 
infinitely large. Under normal circumstances both these events are quite unlikely and contrary to observation. Ample evidence indicates that many populations regulate their numbers by some density dependent mechanism (Tanner, 1966; Mcbridge, 1966).

Density dependent population control can be incorporated into a growth equation by the addition of a damping term modifying the rate of growth as the population increases. One of the many forms that this damping can take is illustrated by the logistic equation

$$
\frac{d x}{d t}=r x(1-x / k)
$$

where $k$, the carrying capacity, is the maximum number of organisms that the environment can support. The logistic equation fits many population growth experiments well enough to be of considerable practical use. Only two constants must be determined, the intrinsic rate of natural increase and the carrying capacity. Neither of the two quantities can be given in general an exact mathematical or biological definition independently of the eq. (3) . Conceptually, the logistic suggests two extremes of evolutionary schemes: (a) evolution toward the fastest possible growth rate: "r-selection"; (b) evolution toward the most efficient use of the environment, resulting in the largest carrying capacity, the "k-selection" (for a discussion see e.g. MacArthur and Wilson, 1967). The utility of the logistic equation 
and of the $r$ or $k$ "selection" concept is limited due to the many simplyfying assumptions underlying the models.

There is no biological justification for the damping term in eq. (3) to vary with the square of population density. Pielou (1977) suggests we can think of quadratic damping as the first approximation in a Taylor expansion of more general density dependences. Inclusion of higher order terms would allow greater flexibility while curve fitting experimental data (Wangersky, 1978) but might also obscure biological insight.

It is biologically likely that actual populations would not respond instantaneously to changes in their numbers as in the logistic equation. Thus, a time lag between the cause and effect should be introduced into single species growth models. A simple model incorporating time delay is due to Hutchinson (1957). A general form of the equation for the model can be obtained by introducing a discrete time lag into the damping term of equation (3).

$$
\frac{d x}{d t}=\operatorname{rx}(1-(x / k))_{t-\tau}
$$

where $\boldsymbol{\tau}$ is the time lag between cause and effect. Several other forms of the differential equation and corresponding difference equations have been proposed (Ross, 1972; Mazanov, 1973; May, 1973, May, 1974). Lefkovitch (1966) has incorporated delayed responses into a matrix algebra for population growth. The inclusion of time delay has a destablizing 
effect; there is an increase in the kinds of solutions possible. Even under stable environments, oscillations can occur which grow large enough to produce extinction in single species populations.

The logistic equation and other deterministic models discussed here display a monotonic approach to an equilibrium population. If they do show oscillatory behavior as in models with time delay, the fluctuations cannot be entirely explained in biological terms. The difficulty with these models is the assumption that populations live under constant environments. All ecological environments show some periodic or random variability, and such fluctuations eventually affect the patterns of growth of a population.

For this reason, the deterministic models of theoretical ecology, though used extensively in demographic work, do not provide adequate estimates or predict future trends of a population. Since most natural communities exist in environments varying randomly over time, the deterministic models need to be modified to include the temporal variability in the fecundity and mortality schedules. We need to consider the effects of environmental unpredictability on population size, the ultimate rate of increase and the asymptotic stable age distribution.

b) The Stochastic Models

The study of population dynamics under stochastic 
environments is more realistic, but often phenomenological considerations lead to mathematically intractable formulations. Perhaps the simplest models take a macroscopic view of the problem and treat population growth as a diffusion process. Rewrite equation (I) as

$$
\frac{d x(t)}{d t}=r(t) x(t)
$$

where the Malthusian parameter, $r(t)$, is now assumed to be a function of time and takes on values consistent with the variability in environment. $r(t)$ can be assumed to be made of two parts

$$
r(t)=\bar{r}+\epsilon(t)
$$

where $\bar{r}$ is a constant mean value of the growth rate and is a stationary stochastic process. Over long time intervals the process $\epsilon(t)$ can be represented in a coarsegrained way by a $\delta$-correlated white noise

$$
\begin{aligned}
\langle\epsilon(t)\rangle & =0 \\
\left\langle\epsilon\left(t_{1}\right) \epsilon\left(t_{2}\right)\right\rangle & =2 \beta \delta\left(t_{2}-t_{1}\right)
\end{aligned}
$$

where brackets denote ensemble averages and $\delta\left(t_{2}-t_{1}\right)$ is the usual Dirac delta function. Here $\beta$ is the spectral density for the random fluctuations. With random perturbations model behavior is more complex than in deterministic models. 
We now have a first order stochastic differential equation; interest now focuses on the probability of observing a population size of $x(t)$ at time $t$ conditional on a known initial size. $x(t)$ must now be viewed as a realization of a continuous and time homogeneous process. Instead of a stable population we now look for a stable probability distribution of a population. We also need to look at the probability of a population going extinct, since the environmental fluctuations may be strong enough to make the ultimate rate of growth less than unity for some realizations.

If the random fluctuations obey equations (5) and (6), the population size behaves as a Markov diffusion process and can be studied in terms of a Wiener process (Capocelli and Ricciardi, 1974; Tuckwell, 1974). The probability density function $f(x(t) \mid x(0))$ for $x(t)$ given initial population, $x(0)$ obeys the Kolmogorov forward equation

$$
\frac{\partial f(x(t) \mid x(0))}{\partial t}=-(\bar{r}+\beta) \frac{\partial}{\partial x}(x f)+\beta \frac{\partial^{2}}{\partial x^{2}}\left(x^{2} f\right)
$$

where $(\bar{x}+\beta) x$ and $2 \beta x^{2}$ are the drift and variance for the process.

A similar transformation of the logistic equation leads to the stochastic differential equation

$$
\frac{d x(t)}{d t}=r(t) x(t)\left(1-\frac{x(t)}{k}\right), 0 \leqslant x(t) \leqslant \infty
$$

This equation has been studied by Levins (1969) and May 
(1973); Tuckwell (1974) transforms it into an ornsteinUhlenbeck process. Results can be immediately obtained for the probability distribution and the extinction probabilities since the process is fully understood analytically. Capocelli and Ricciardi (1974) have derived expressions for the expected first passage time $\mathrm{T}$ at some level $\mathrm{S}$ and its variance $\sigma^{2}(S, X(0))$

$$
\begin{aligned}
& T(s, x(0))=|\ln (x(0) / S)| /|\bar{r}| \\
& \sigma^{2}(s, x(0))=\left(2 \beta / \bar{r}^{2}\right)|\ln (x(0) / s) / \bar{r}|
\end{aligned}
$$

also the most likely value $t$ for the first passage time is

$$
\tau=\left\{\begin{array}{l}
\ln ^{2}(x(0) / s) / 6 \beta, \bar{r}=0 \\
-3 / 2+\left[(9 / 4)+(\bar{r} / 2 \beta)^{2} \ln (x(0) / 5)\right]^{1 / 2}, \bar{r}_{\neq 0}
\end{array}\right.
$$

The use of diffusion approximations in the study of ecological models is mathematically convenient but not very realistic. It implies that both death and birth are continuous processes. It also assumes that a gross average rate of growth over the life span of an individual may be meaningfully deduced from the data. Both these assumptions seriously limit the applicability of the model. We know that birth and death processes are discrete and would be more accurately described by a stochastic difference equation. It is also reasonable to assume that the fertilities 
and the survival rates and hence the growth rate of a population is a function of age or size distribution. These considerations lead us to Leslie's model discussed in the next chapter. 


\section{CHAPTER III}

\section{LESIIE'S MODEL}

Earlier we have discussed the need to work with a model that employs a discrete time variable and age structure, giving due weights to the age-specific survivals and mortalities. This approach is also preferable because most available data is in the discrete form and because it coincides with the actuarial practice. Leslie's model is the best known and most detailed of such models.

The Leslie model (1945) considers only one-sex (usually females) in a single closed population divided into age groups corresponding to a discrete unit interval of time. A decision as to the best choice of a unit time interval is usually dictated by the available data. Pollard (1973) shows that there is little difference in the deterministic asymptotic results when different age groupings are used in the case of human populations. The results may not be as satisfactory for other organisms. The model is best suited to a matrix formulation. The general form of a Leslie matrix is

$$
L=\left[\begin{array}{ll}
A & 0 \\
B & C
\end{array}\right]
$$


The first row in $A, a k x$ matrix, contains fertilities $m(i) \quad 0, i=1,2, \ldots . k$ with $m(k)$ having the last non-zero value. The survival rates for the first $k$ age-classes are on the off-diagonal as indicated:

$$
\underline{A}=\left[\begin{array}{ccccc}
m(1) & m(2) & m(3) & \ldots & m(k) \\
p(1) & 0 & 0 & \ldots & 0 \\
0 & p(2) & 0 & \ldots & 0 \\
\vdots & & & & \\
0 & \ldots & 0 & p(k-1) & 0
\end{array}\right]
$$

A is non-negative, irreducible since $m(k)$ does not vanish Sykes (1969) and primitive if the greatest common divisor of the indices of the positive elements in the first row is one (Rosenblatt, 1957). Also A satisfies the hypotheses of the Perron-Frobenius theory of non-negative matrices. According to this theory maxtrix $\mathrm{A}$ has a positive dominant eigenvalue $\lambda_{\circ}$ of multiplicity one corresponding to some column eigenvector $U$ and a row eigenvector $V^{\prime}$, both of which have positive elements; further $\lambda_{0}$ is greater in absolute size than any other eigenvalue. An excellent account of the properties of $A$ is given by parlett (1970).

It is well known that $\underline{A}$ contains all the important information about $\underline{I}$. For example, the age groups beyond reproduction do not contribute to the growth rate of the whole population. Hence, without loss of generality $\underline{A}$ can 
be used to work out the mechanics of the Leslie model. Consider the female population in the first $\mathrm{k}$ age groups arranged in the form of a $k$-dimensional vector $\mathrm{N}_{t}$ at any time $t$. At the end of each time interval $(t, t+1]$, $t=0,1,2, \ldots$ a new population vector $N_{t+1}$ is determined by the equation

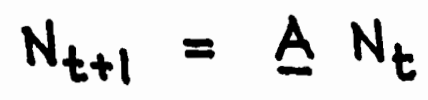

Iteration of the above equation yields

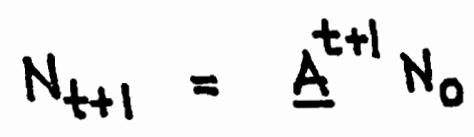

where $\mathrm{N}_{0}$ is the initial population structure. Since $\underline{A}$ has a positive dominant eigenvalue $\lambda_{0}$, largest in absolute size, it eventually swamps the effects of other eigenvalues while any initial age distribution asymptotically approaches $U$, the stable age distribution. Pielou (1969) illustrates the ergodic property nicely in a graph for population trajectories belonging to different Leslie matrices with the same $\lambda_{0}$. They both converge to the same stable age distribution though they choose different paths depending on higher eigenvalues.

Within the foregoing deterministic description we can incorporate the temporal variability of the environment quite naturally into Leslie theory. Fertilities and survival rates are considered as functions of time. At each time interval $(t, t+1], t=1,2, \ldots$ fertilities $m_{t}(i), i=1,2, \ldots, k$ 
and survival rates $p_{t}(i), i=1,2, \ldots, k$ are contained in a Leslie matrix $\underline{x}_{t}$. For any realization of the process the successive Leslie matrices are chosen from a random set to form a Markov sequence of environmental states such that

$$
N_{t+1}=\underline{x}_{t} N_{t}=\underline{x}_{t} \underline{x}_{t-1} \ldots \underline{x}_{0} N_{0}
$$

Total population at any time $t$ is

$$
M_{t}=\sum_{i=1}^{k} N_{t}(i)
$$

Define the cummulative growth in total population number in time $t$ to be

$$
\Lambda_{t}=\frac{M_{t}}{M_{0}}
$$

where $\mathrm{M}_{\mathrm{O}}$ is initial fixed population corresponding to $\mathrm{N}_{0}$. Also since this is a geometric growth model, define the ultimate rate of increase as $\lim _{t \rightarrow \infty} 1 / t \ln \Lambda_{t}$. Boyce (1977) has studied the long run growth rates numerically. Cohen (1977) has established the demographic weak ergodic theorm for Markov sequences of vital rates. Tuljapurkar and Orzack (1980) have extended earlier results and looked at the long run distribution of growth rates in fluctuating environments. They establish that the logrithm of the cummulative growth in number is asymptotically normally distributed at long times

$$
\ln \Lambda_{t} \underset{t \rightarrow \infty}{\longrightarrow} \sim N\left(a t, \sigma^{2} t\right)
$$


where $a, \sigma^{2}$ are the scaled mean and variance respectively. They have employed the lognormality of cummulative growth rate to explore the probability of extinction analytically and numerically. 


\section{CHAPTER IV}

BOUNDS FOR POPULATION GROWTH RATE AND AGE STRUCTURE IN VARIABLE ENVIRONMENTS

Assuming for any given environment a reasonable estimate can be made as to the span of variability of each fertility and survival rate for all times such that

$$
\begin{aligned}
& P_{\min }(i) \leqslant P_{t}(i) \leqslant P_{\max }(i) \\
& m_{\min }(i) \leqslant m_{t}(i) \leqslant m_{\max }(i)
\end{aligned}
$$

Using these bounds on fertilities and survivals we can construct matrices $x_{\max } x_{\min }$. Then elementwise

$$
\underline{X}_{\min } \leqslant \underline{X}_{t} \leqslant \underline{X}_{\max } ; \text { for all } t
$$

Using equation (10) it can be shown by iteration

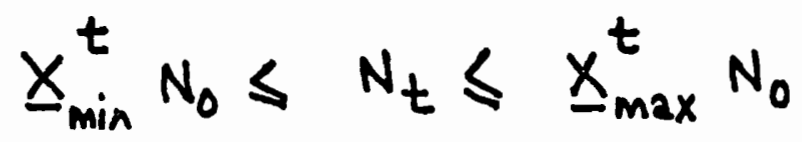

Since the inequality is true elementwise, using equations

(11) and (12)

$$
\lim _{t \rightarrow \infty} \frac{1}{t} \ln \Lambda_{\min } \leqslant \lim _{t \rightarrow \infty} \frac{1}{t} \ln \Lambda_{t}=a \leqslant \lim _{t \rightarrow \infty} \frac{1}{t} \ln \Lambda_{\max }
$$

corresponds to a constant matrix involving $P_{\min }(i)$ and $\min (i)$. From deterministic theory $\mathrm{N}_{t} \lambda_{\min }^{t}$ No where $\min$ is the dominant eigenvalue of $x_{\min }$ and the logrithmic growth rate for the process is $\lim _{t \rightarrow \infty} \frac{1}{t} \ln \Lambda_{\min }=\ln \lambda_{\min }$. similarly 
19

$\lim _{t \rightarrow \infty} \hbar^{\ln } \Lambda_{\text {max }}=\ln \lambda_{\text {max }}$. This shows that the long run distribution of logrithmic growth rate is bound by the extremes of values taken by survivals and fertilities. Also, the eventual rate of growth would lie somewhere between $\lambda_{\min }$ and $\lambda_{\max }$.

Another useful inequality provides the bounds on age structure. Consider a Leslie matrix for which all entries are constant except $p_{t}(1)=p . p$ is a random variable with probability density $W(p)$ and bounded by constants $p_{1}$ and $p_{2}$, $p_{1} \leqslant p(t) \leqslant p_{2}$. Define $U_{t}=\frac{N_{t}(2)}{N_{t}(1)}, t=0,1,2 \ldots$ the ratio of the second age class to the first at time $t$. The choice of a new matrix at the end of a time interval $(t, t+1]$ is in effect a choice of a new $p$. We can write

$$
\begin{aligned}
u_{t+1} & =f_{p}\left(u_{t}\right) \\
& =f_{p}\left(f_{p}\left(\ldots f_{p}\left(u_{0}\right) \ldots\right)\right)
\end{aligned}
$$

where $f_{p}(u)$ is some function of $p$ and $v_{t}$ determined by the equation for the model. If $U_{0}$ at $t=0$ is known then from the bounds on $p$ it can be deduced

$$
f_{p_{1}}\left(u_{0}\right) \leqslant f_{p}\left(u_{0}\right) \leqslant f_{p_{2}}\left(u_{0}\right)
$$

and

$$
f_{p_{1}}\left(f_{p_{1}}\left(u_{0}\right)\right) \leqslant f_{p}\left(f_{p}\left(u_{0}\right)\right) \leqslant f_{p_{2}}\left(f_{p_{2}}\left(u_{0}\right)\right)
$$

etc. 
If we fix $p=c$, a constant then $N_{t} \rightarrow \lambda_{c}^{t} u$ where $\lambda_{c}$ is the dominant eigenvalue and $U$ is the corresponding right eigenvector. After long time we have a stable age distribution which implies $U_{t} \rightarrow U_{c^{\prime}}$ a constant. Applying this to equation (18) we have

(20)

$$
\mathrm{u}_{\mathrm{p}_{1}} \leqslant \mathrm{U}_{\mathrm{t}} \leqslant \mathrm{u}_{\mathrm{p}_{2}}, \text { for all } t
$$




\section{CHAPTER V}

\section{A TWO DIMENSIONAL EXAMPLE}

Let the survival from first to the second age class be a random variable chosen from some distribution $w(p)$. The basic equation is:

$$
\left[\begin{array}{l}
N_{t+1}(1) \\
N_{t+1}(2)
\end{array}\right]=\left[\begin{array}{cc}
m(1) & m(2) \\
p & 0
\end{array}\right]\left[\begin{array}{l}
N_{t}(1) \\
N_{t}(2)
\end{array}\right]
$$

Here $m(1)$ and $m(2)$ are fertilities of the first and the second age classes and $p$ is the survival rate from the first to the second age class. Define $y_{t}(1)=N_{t}(1) / M_{t}$ and $y_{t}(2)=N_{t}(2) / M_{t^{\prime}}$ the fractions of population in the two age classes and $U_{t}=\frac{N_{t}(2)}{N_{t}(1)}$ as their ratio.

Numerical simulations of such a model to explore the convergence to lognormality and the effects of environmental variance and auto correlation have been reported by Tuljapurkar and orzack (1980). Here we study the following: Starting from a known distribution $w(p)$ of the survival parameter $p$ how are $y_{t}(i)$ and $U_{t}$ distributed after long times? How is the variance in $\mathrm{p}$ related to the variance in $y_{t}$ and $U_{t}$ ? How is the average of $y_{t}$ related to average value of $p$ ? For $m(1)=.85, m(2)=1.0$ and $w(p) \sim \operatorname{triangular}(\beta)$ with 
$\bar{p}=0.4$ Monte Carlo simulations were done on a computer. 500 population trajectories were calculated for the process, each one being 500 generations long. For these trajectories the parameters $y_{t}, U_{t}$ were calculated at $t=500$ and their histograms were plotted. The triangular distribution for $p$ was chosen because it is compact as compared to normal and can be generated more efficiently on a computer. The method to generate the triangular distribution and a Fortran program for the process is given in Appendix A. We find a triangular distribution also results for $y_{t}$ and $U_{t}$ (see Figures $1-3$ ).

To study the variance and average of $y_{t}$ and $U_{t^{\prime}}$ calculations were made for a number of different values of $\beta$ about a fixed mean. Figure 4 illustrates the relationship between the variance in $p$ and in $y_{t}(1)$ : An increasing variance in $p$ increases the variance observed in $y_{t}(1)$. The results are similar for $y_{t}(2)$ and $U_{t}$. With $E(p)$ $(E(\cdot)$ denotes expectation) held fixed, changes in var ( $p)$ did not affect the position of the means of $y_{t}$ and $U_{t}$. Also, $E\left(U_{t}\right)$ and $E\left(y_{t}\right)$ are found to be in excellent agreement with what one would expect from the deterministic theory if $\mathrm{p}$ is replaced by its average value.

We also looked at the problem of age structure analytically. From equations (18) and (21) we have

$$
f_{p}(u)=\frac{p}{m_{1}+m_{2} u}=u^{\prime}, \text { say }
$$




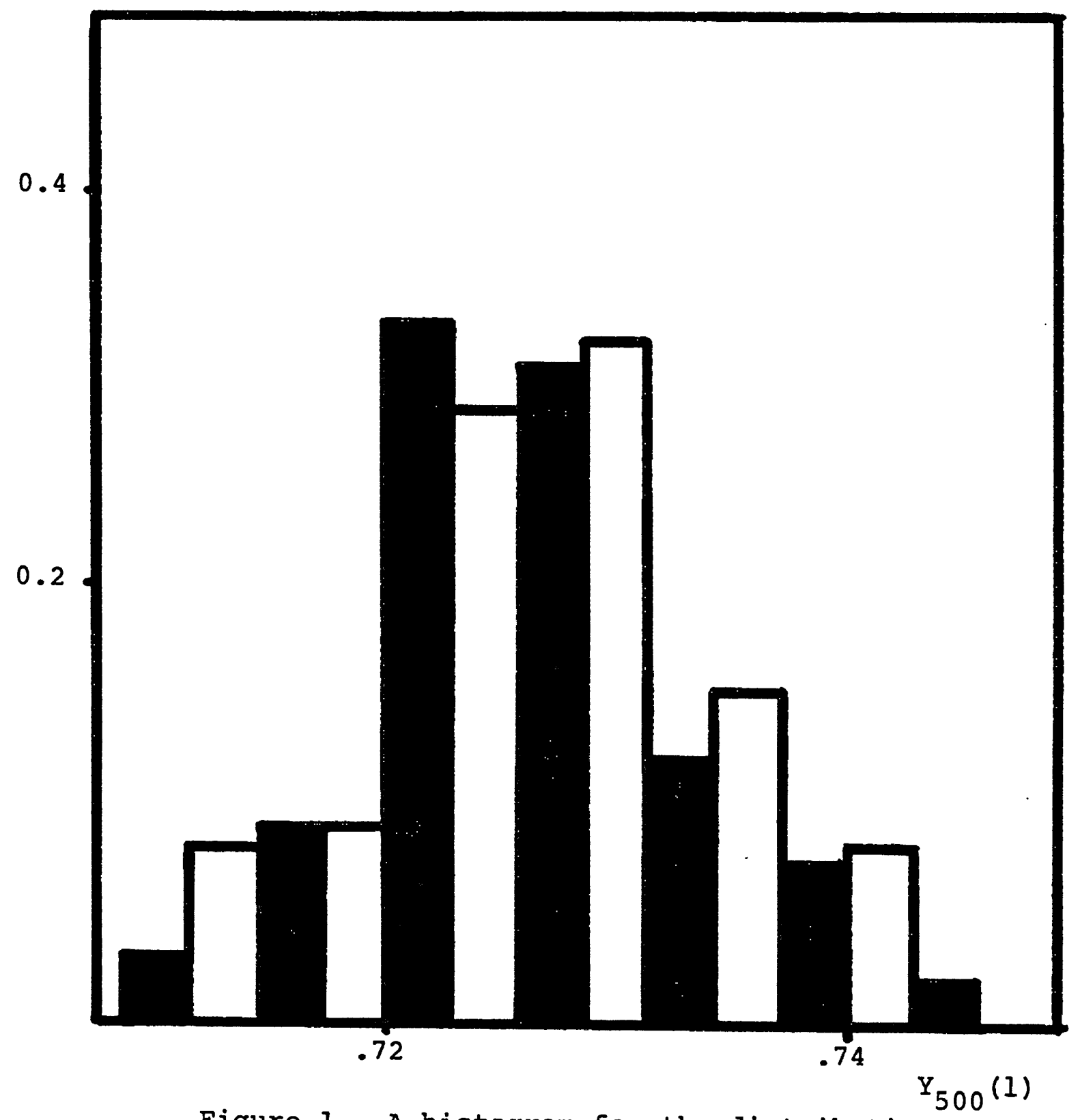

Figure 1. A histogram for the distribution

of $Y_{500}(1)$, the fraction of population is the ilrst age class when the survival rate is distributed triangular (.02). 


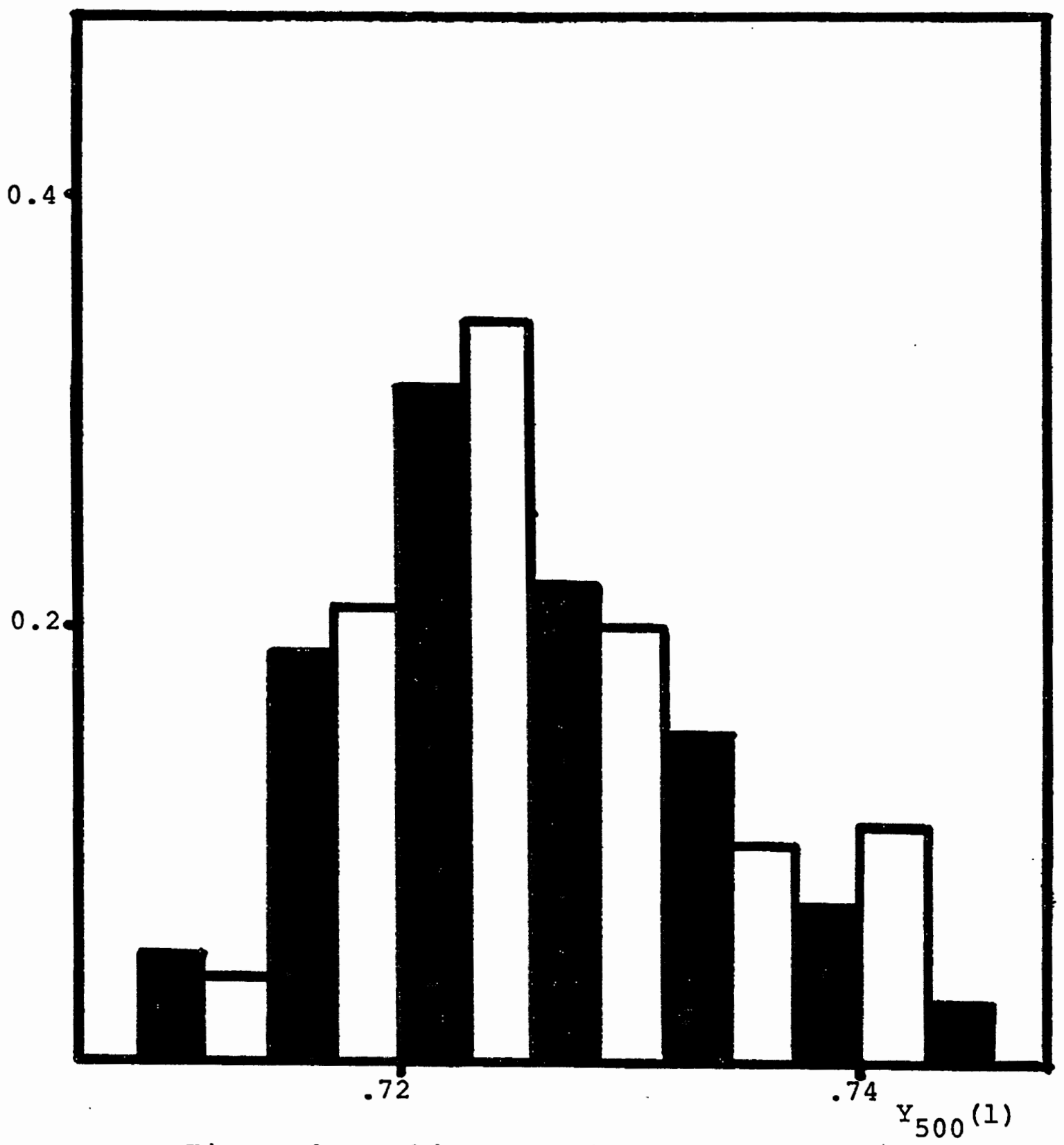

Figure 2. A histogram for the distribution of Y 500 (1), the fraction of population is the first age class when the survival rate is distributed $\sim$ triangular (.10). 


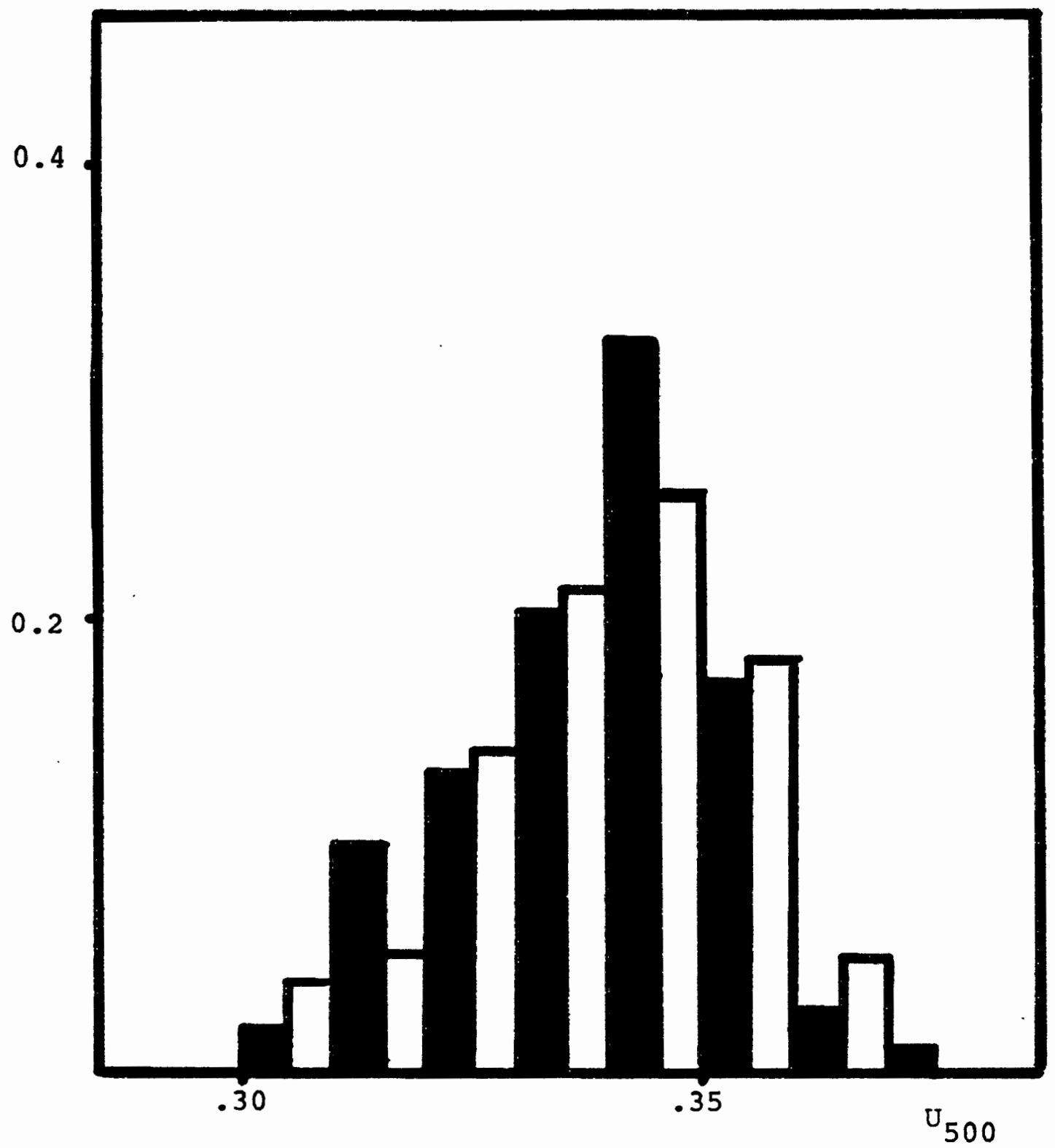

Figure 3. Distribution for $\mathrm{U}_{500^{\prime}}$ the ratio of the second age class the first, when the survival rate is distributed triangular (0.1). 


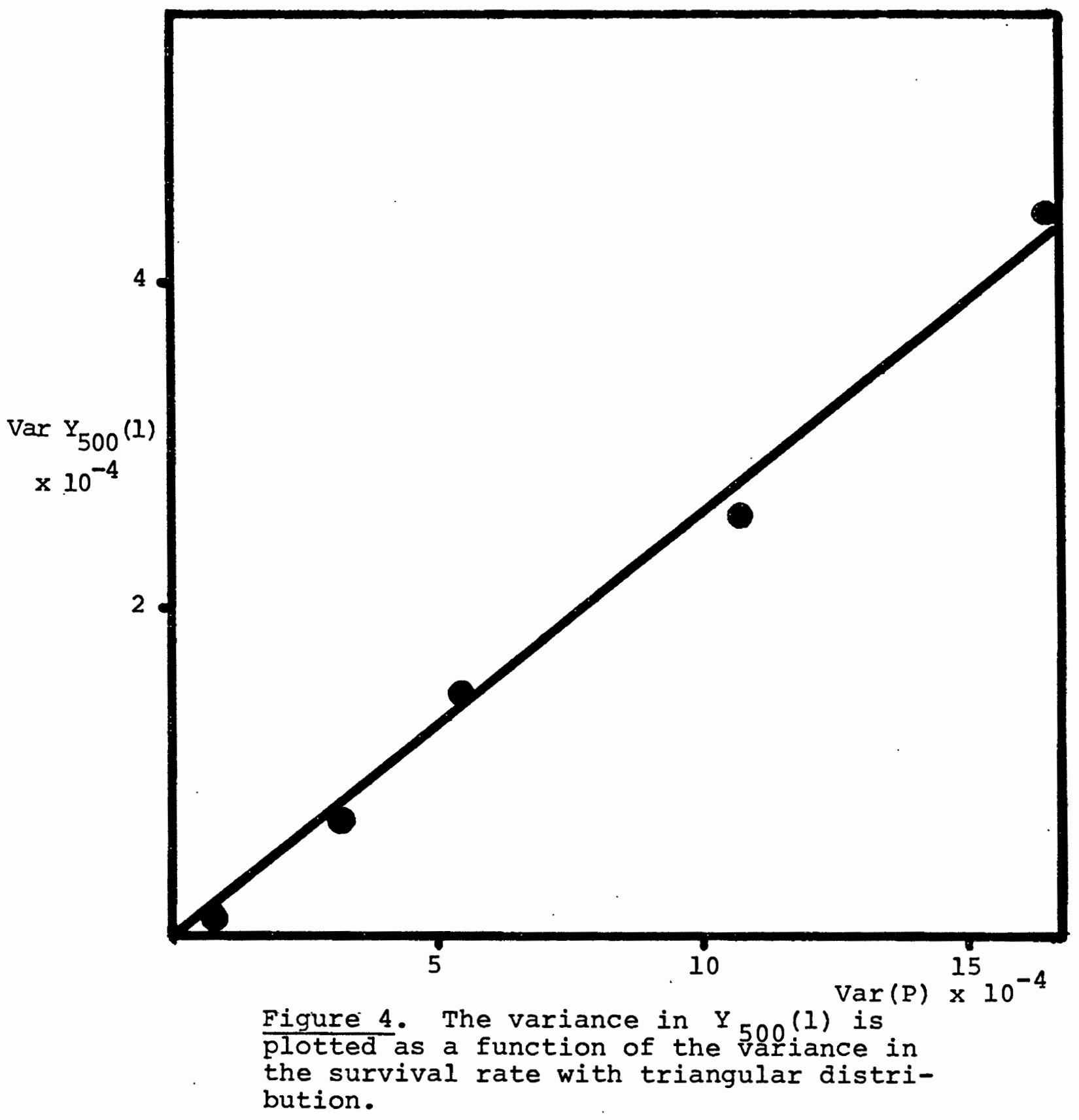


27

then

$$
u=f_{p}^{-1}\left(u^{\prime}\right)=g_{p}\left(u^{\prime}\right)=\frac{p}{m_{2} u^{\prime}}-\frac{m_{1}}{m_{2}}
$$

Let the stationary distribution of $U$ be

$h(\eta) d \eta=\operatorname{Prob}\left\{u \in(\eta, \eta+d \eta)\right.$ to get the distribution of $U^{\prime}$ as $h(\xi) d \xi=\operatorname{Prob}\left\{u^{\prime} \in(\xi, \xi+d \xi)\right.$. Then we have

$$
h(\xi) d \xi=\int_{I} d p w(p) h(\eta) d \eta
$$

where I means that for each $p$ we use that $\eta$ which sarisfits $\xi=f_{p}(\eta)$. So on R.H.S. of equation (24)

$$
h(\xi)=\int d p \omega(p) h\left(g_{p}(\xi)\right) \frac{p}{b \xi^{2}}, \xi \text { is fixed on R.HS. }
$$

Rewriting (25) after a change of variables $\eta=g_{p}(\xi)$, $p=\left(m_{1}+m_{2} \eta\right) \xi, \quad d p=b \xi d \eta$

$$
h(\xi)=\int \omega[\xi(a+b \eta)] h(\eta)(a+b \eta) d \eta
$$

A relatively simple result can be obtained if $p$ is uniformly distributed, since then $w(p)=$ constant. As $\int h(\xi) d \xi=1$ we have

$$
h(\xi)=\frac{1}{u_{p_{2}}-u_{p_{1}}}
$$


If $\mathrm{p}$ belongs to other more complex distributions, the analytical results are not easily extended and it may be necessary to look for a numerical solution to the problem. To investigate the stationary probability distribution numerically, a Markov matrix with row sums equal to unity is constructed by considering a mapping of $U_{t}$ on $U$ - The alogrithm for the process and its implementation in Fortran are given in the Appendex B. As an example we take $p$ distributed uniform on $(\cdot 3, \cdot 5)$ with other parameters having the same values as described earlier. As expected the results show a uniform distribution for $U_{t}$ within the analytical range of $v_{t}$ obtained in the last section.

\section{RESULTS}

In summary, we find the following: (I) the age structure distribution closely follows the distribution of the random variable in the Leslie matrix; this random variable is the survival rate in the above two dimensional examples. (2) the variance in age structure is a linear function of the variance in the random variable and (3) the mean agestructure is tied to the predicted deterministic age structure for the average value of the random variable. We expect the results would be true for higher dimensional models. 
CHAPTER VI

SIMULATION IN THREE DIMENSIONS

A more efficient and faster alogrithm is developed to calculate the population trajectories and relevant statistics on a computer. The process is viewed as a sequence of births in time. Numbers in higher age classes can be computed using the relevant survival rates. Define

$$
\psi_{1}=1=\psi_{2} ; \quad \psi_{3}=p_{2}, \ldots, \psi_{i}=\prod_{2}^{i-1} p_{i}
$$

and

$$
\begin{aligned}
& x_{i}=m_{i} \psi_{i} \\
& z_{1}=1 ; z_{2}=p_{1}(t-1), \ldots, z_{i}=p_{1}(t-i+1)
\end{aligned}
$$

Then the number of births $B(t)$ at time $t$ can be written as

$$
N_{t}(1)=B(t)=\sum_{i=1}^{k} X_{i} Z_{i} B(t-i)
$$

The above equation assumes the variability in environment affects only the first survival rate. We can write a simplar equation for $B(t)$ when other parameters show random variations. 
The equation can be iterated to yield a sequence of births of appropriate length provided the births at the first $k$ intervals could be supplied. The latter condition is no more restrictive than the specification of an initial age distribution vector $\mathrm{N}_{0}$ in the matrix multiplication method. In any case, due to the ergodic property of the process, it is not critical what values are chosen for the first $k$ births.

To work with the equation $(28)$ we need to store
$\{B(t)\}_{t}^{t-k},\left\{X_{k}\right\}_{1}^{k}$, and $\left\{Z_{i}\right\}_{1}^{k-1} ; 3 k$ quantities in all. Iteration, requires $2 \mathrm{k}$ multiplies and $\mathrm{k}$ adds in each step. This scheme saves substantial storage space and operations. By contrast if matrix methods are employed we need to store $k^{2}+k$ quantities ( $a k^{2}$ matrix and a $k$ vector) and require $k^{2}$ multiplies and $k(k-1)$ adds in each step.

We also note that the result obtained by Tuljapurkar and orzack (1980) for the lognormality of $t$ can be easily extended for the birth sequence. Their proof is strict in the sense that the process converges to lognormality elementwise as $t \rightarrow \infty$. Hence, we can conveniently write for the logrithmic growth rate

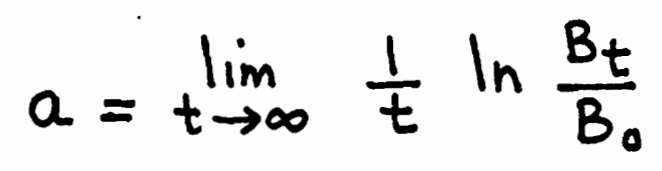

We used this alogrithm for numerical simulations in three dimensions. The main purpose of the study was to 
look at the logrithmic expectation and variance of the growth rate in relation to the extent of fluctuations. Also, a numerical test of the analytical results reported by Tuljapurkar (1981) was sought.

In time populations may decline and finally may disappear if the environmental fluctuations are too strong. A stochastic model should be able to predict the possibility of such an event, i.e., the probability of extinction. Since $\mathrm{N}_{0}$ has nonnegative components in our model, $\mathrm{N}_{t}$ and $M_{t}$ would always be positive however small (Keiding, 1975). The problem is avoided by defining an extinction level, a pre-assigned number at which populations can be considered extinct. We explore this problem numerically.

\section{EXAMPLE}

We study an example in three dimensions due to Boyce (1977) using the alogrithm developed earlier. Here $m(1)=0$, $m(2)=1.5, m(3)=2.2$ are the fertilities for the three age classes. The survival rate for the second to the third age class $\mathrm{p}_{2}=0.25$ is a constant. The survival from the first to the second age class incorporates the random variability of the environment in $p(1)=.6 \pm \epsilon_{1}$, where $\epsilon_{1}$ is uniformly distributed on $(-\beta, \beta)$ with no serial correlation. Also, the number of births at the first three time intervals is: $B(0)=1000, B(1)=1100, B(2)=1200$. A program for the process is given in the Appendix $C$. 
A sequence of births for 500 time intervals was calculated. A random number generator $\sim U(0,1)$ was first used to decide the sign of variation in $p_{1}$ and again for the magnitude of variation. Various statistics were calculated using 200 such birth sequences. Calculations were made for several different $\beta$. Estimates of errors were made by doing the experiment a large number of times. Another set of calculations was made for a given $\beta$ and $E\left(p_{1}\right)$ but different values of $\mathrm{m}(3)$. Test calculations were also made for Leslie models appropriate for fish populations with large fecundity $\left(10^{5}\right.$ ) and very small survival of the first age class $\left(10^{-5}\right.$ ) with essentially the same results.

\section{RESULTS}

(1) An increasing value of parameter $\beta$ decreases the logrithmic average, $a$ and increases the logrithmic variance, $\sigma^{2}$. A graph between $a$ and $\sigma^{2}$ (Figure 5) shows that the two parameters are linearly related. The intercept on the a- axis provides the expected deterministic result for the logrithmic growth rate.

(2) If $\beta$ is held fixed and different values are assigned to $\mathrm{m}(3)$; we obtain a system of parallel lines for different average values of $p_{1}$ in a plot of a vs $\sigma^{2}$. It shows that a decreasing logrithmic average whether it is due to larger fluctuations or a decreasing fertility increases the logrithmic variance. 
(3) The values of a obtained from simulations for small $\beta$ are remarkably close to the calculated values when the analytical formula reported by Tuljapurkar (1981) is used. We find the formula over-estimates for larger values of $\beta$; as much as by 508 when $\beta$ is as large as $E\left(p_{1}\right)$. The formulae for variance in Tuljapurkar (1981) provides equally good results for small perturbations; for higher perturbations it underestimates the variance (see. Table I).

(4) The probability of extinction at any level (see Figure 7 for numerical values) for a population trajectory decreases rapidly for a given environment with time: The maximum number of births remain fairly stable with increasing $\beta$, i.e., some populations grow large even under severe conditions and escape extinction. 


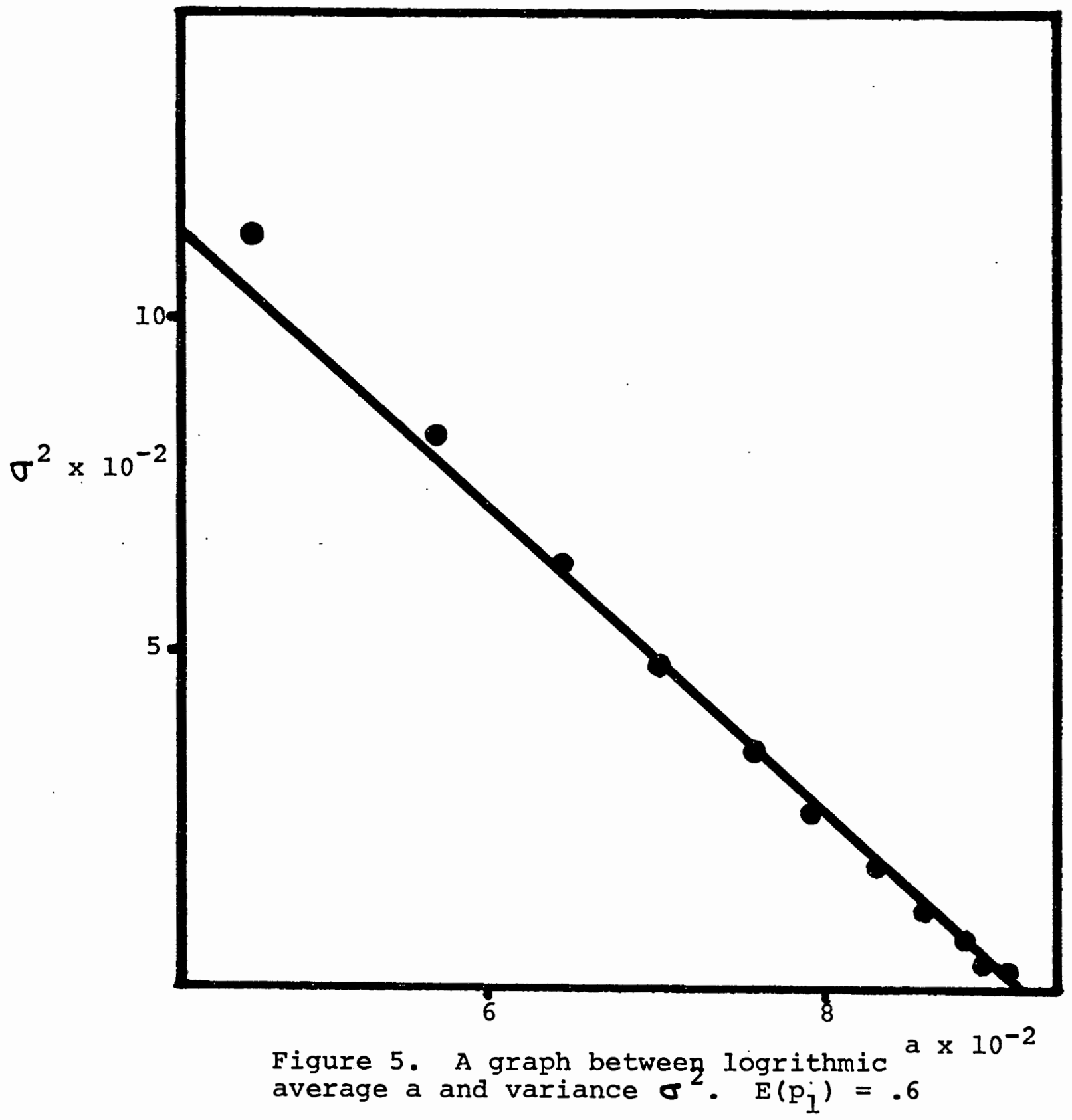




\section{TABLE I}

COMPARISON OF NUMERICAL AND ANALYTICAL VALUES OF a AND $\sigma^{2}$

$\begin{array}{ccccc}\begin{array}{c}\text { Magnitude of } \\ \text { Fluctuation } \\ (\beta)\end{array} & a \times 10^{-1} & \sigma^{2} \times 10^{-2} & a \times 10^{-1} \sigma^{2} \times 10^{-2} \\ 0.025 & 0.914 & 0.011 & 0.915 & 0.004 \\ 0.050 & 0.912 & 0.041 & 0.914 & 0.015 \\ 0.075 & 0.911 & 0.105 & 0.912 & 0.038 \\ 0.100 & 0.907 & 0.179 & 0.910 & 0.060 \\ 0.150 & 0.896 & 0.410 & 0.902 & 0.135 \\ 0.200 & 0.880 & 0.748 & 0.892 & 0.240 \\ 0.300 & 0.832 & 1.80 & 0.862 & 0.540 \\ 0.400 & 0.756 & 3.52 & 0.820 & 0.961 \\ 0.500 & 0.643 & 6.31 & 0.766 & 1.50\end{array}$

1. Numerical values are obtained with $E\left(p_{1}\right)=0.6, m(1)=0$, $\mathrm{m}(2)=1.5, \mathrm{~m}(3)=2.2$ and $\mathrm{p}_{2}=0.25$ corresponding analytical values are calculated using formulas reported in Tuljapurkar (1981).

and

$$
a=\ln \lambda_{0}+\frac{\tau^{2}}{2 \lambda_{0}}
$$

$$
\sigma^{2}=\frac{\tau^{2}}{2 \lambda_{0}}
$$




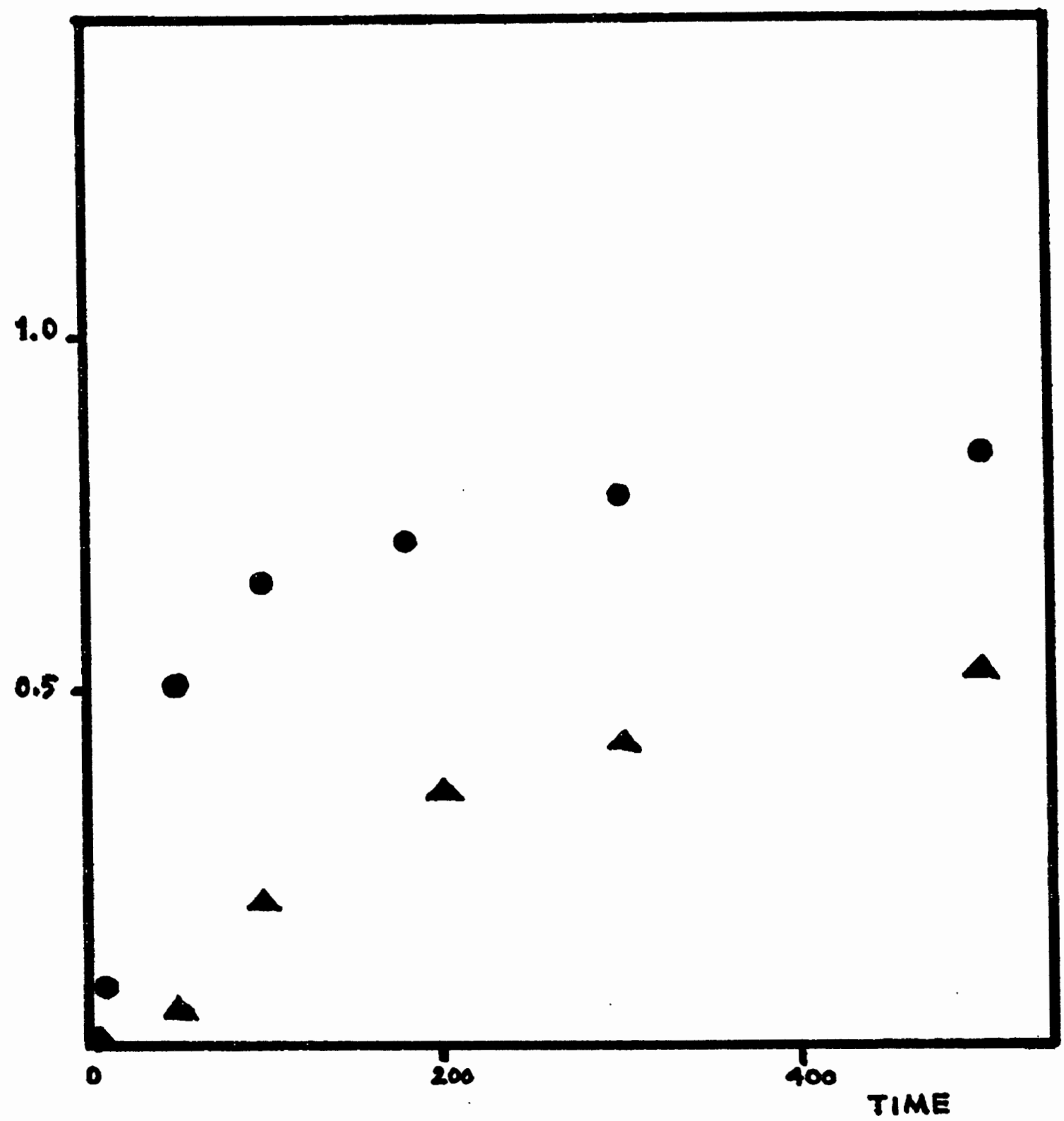

Figure 7. Extinction probability as a

function of time. Here $E\left(p_{1}\right)=\cdot 5, \beta=\cdot 3$. Extinction boundary for is 500 births, for 100 births. 
REFERENCES

Athreya, K. B. and Karlin, S. 1971. On Branching Processes with Random Environments, I: Extinction Probabilities. Ann. Math. Stat. 42:1499-1520.

Bernardelli, H. 1941. Population Waves. J. Burma. Res. Soc. $31: 1-18$.

Boyce, M. S. 1977. Population Growth with Stochastic Fluctuations in the Iife Table. Theo. Pop. Biol. $12: 366-373$.

Capocelli, R. M. and Ricciardi, L. M. 1974. A Diffusion Model for Pop. Growth in Random Environment. Theo. Pop. Biol. 5: 28-41.

Coale, A. J. 1972. The Growth and Structure of Human Populations: A Mathematical Investigation. Princeton University Press, Princeton, N. J.

Cohen, J. E. 1976. Ergodicity of Age Structure in Populations with Markovian Vital Rates I. Countable States. J. Amer. Stat. Assoc: 71:335-339.

Cohen, J. E. 1977a. Ergodicity of Age Structure in Populations with Markovian Vital Rates II. General States. Adv. Appl. Prob. 9:18-37.

Cohen, J. E. 1977b. Ergodicity of Age Structure in Populations with Markovian Vital rates III. Finite State Moments and Growth Rate; an Illustration. Adv. Appl. Prob. 9: $469-475$.

Gourley, R. S. and Lawrence, C. E. 1977. Stable Population Analysis in Periodic Environments. Theo. Pop. Biol. 11: $49-59$.

Hutchinson, G. E. 1957. Concluding Remarks. Cold Spring Harbor Symp. Quart. Biol. 22:415-427.

Keiding, N. 1975. Extinction and Exponential Growth in Random Environments. Theo. Pop. Biol. $\underline{8}: 49-63$.

Keyfitz, N. 1968. Introduction to Mathematics of Populations. Addison-Wesley, Reading, Mass. 
Lefkovitch, I. P. 1966. A population Growth Model Incorporating Delayed Responses. Bull. Math. Biophys. 28:219-233.

Leslie, P. H. 1945. On the Use of Matrices in Certain Population Mathematics. Biometrika 35:213-245.

Levins, R. 1969. The Effect of Random Variations of Different Types on Population Growth. Proc. Nat. Acad. Sci. 62:1056-1060.

Lewis, E. G. 1942. On the Generation and Growth of a Population. Sankhya 6:93-96.

Lopez, A. 1961. Problems in Stable Population Theory. office of Population Research, Princeton University Press, Princeton, N. J.

Lotka, A. J. 1907. Relation Between Birth Rates and Death Rates. Science, new series 26:21-22.

MacArthur, R. H. and Wilson, E. O. 1967. Island Biogeography. Princeton, N. J., Princeton University Press, p. 203.

MacArthur, R. H. 1968. Selection for Iife Tables in Periodic Environments. Amer. Natur. 102:381-383.

Malthus, T. 1798. An Essay on the Principle of Population. Printed for $J$. Johnson in St. Paul's Churchyard, Iondon.

May, R. M. 1973. On Relationships Among Various Types of Population Módels. Am. Nat. 107:46-57.

May, R. 1973. Stability and Complexity in Model Ecosystems. Princeton Univ. Press, Princeton, N. J.

May, R. M. 1974. Biological Populations with Nonoverlapping Generations: Stable Points, stable Cycles, and Chaos. Science 186:645-647.

Mazanov, A. 1973. On Difference - Difference Growth Equation. Search $\underline{4}: 199-201$.

McBride, G. 1966. The Conflict of Crowding. Discovery $27: 16-19$.

Namkoong, G. 1972. Persistence of Variances for Stochastic, Discrete Time Population Growth Models. Theo. Pop. Biol. 3: $507-518$. 
Parlett, B. 1970. Ergodic Properties of Populations I: The One Sex Model. Theo. Pop. Biol. 1:191-207.

Pielou, E. C. 1977. Mathematical Ecology. John Wiley and Sons, New York.

Pollard, J. H. 1973. Mathematical Models for the Growth of Human Populations. Cambridge Univ. Press, New York.

Rosenblatt, D. 1957. On the Graphs and Asymptotic Forms of Finite Boolean Relation Matrices and Stochastic Matrices, Naval Res. Logist. Quart. 4:151-167.

Ross, G. G. 1972. A Difference Differential Model in Population Dynamics. J. Theo. Biol. 37:477-492.

Sharpe, F. R. and Lotka, A. J. 1911. A Problem in Age Distribution. Philosophical Magazine 21:435-438.

Skellam, J. G. 1967. Seasonal Periodicity in Theoretical Population Ecology. Proc. of the 5th Berkeley Symp. in Math. Stat. and Prob. (I. LeCam and J. Neyman, Eds.), Vol. 4, pp. 179-205, Univ. of California Press, Berkeley.

Sykes, Z. M. 1969. Some Stochastic Versions of the Matrix Models for Population Dynamics. J. Amer. Stat. Assoc. 64: $111-130$.

Sykes, Z. M. 1969. On Discrete Stable Population Theory. Biometerics 25 .

Tanner, J. T. 1966. Effects of Pop. Density on Growth Rates of Animal Pop. Ecology 47:733-45.

Tuckwell, H. C. 1974. A Study of Some Diffusion Models of Population Growth. Theo. Pop. Biol. 5:345-357.

Tuljapurkar, S. D. 1981. Population Dynamics in Variable Environments II. Correlated Environments, Sensitivity Analysis and Dynamics. Submitted: Theo. Pop. Biol.

Tuljapurkar, S. D. and Orzack, S. H. 1980. Population Dynamics in Variable Environments $I$. Long Run Growth Rates and Extinction. Theo. Pop. Biol. 18:314-342.

Volterra, $V$. 1926. Variazioni e fluttuazioni del numero d'individui in specie animali conviventi. Med. Acad. Lincei.: 2:31-113 (Translation in an appendix to Chapman's Animal Ecology. New York, 1931). 
Volterra, V. 1937. Principes de biologie mathematique. Acta Biotheoretica $\underline{3}: 1-36$.

Wangersky, P. J. 1978. Lotka-Volterra Population Models. Ann. Rev. Ecol. Syst. 1978. 9:189-218.

Weissner, E. W. 1971. Multitype Branching Processes in Random Environments. J. Appl. Prob. 8:17-31. 
APPENDIX A

ALOGRITHM FOR TRIANGULAR DISTRIBUTION AND FORTRAN PROGRAM FOR STATIONARY DISTRIBUTION

For a triangular distribution

$$
f(z) d z=\operatorname{Prob}\{z \in(z, z+d z)
$$

where

with

$$
\begin{aligned}
& f(z)= \begin{cases}1 / \beta(1+z / \beta), & z \leqslant 0 \\
1 / \beta(1-z / \beta), & z \geqslant 0\end{cases} \\
& -\beta \leqslant z \leqslant \beta
\end{aligned}
$$

The cumulative distribution function is

$$
F(z)=\left\{\begin{array}{cc}
(z+\beta)^{2} / 2 \beta^{2}, & z \leqslant 0 \\
1-(\beta-z)^{2} / 2 \beta^{2}, & z \geqslant 0
\end{array}\right.
$$

The mean and variance are zero and $\beta^{2} / 6$ respectively. We note the following theorm without proof before we describe the alogrithm.

Theorm

Let $z$ be any random variable with cumulative distribution function $F(x)=p[z \leqslant x]$. Let $U$ be a random variable 
uniformly distributed $(0,1)$. Then we can write $z=F^{-1}(U)$. In the case of triangular distribution, we have

(AI)

$$
F^{-1}(u)= \begin{cases}\beta(\sqrt{2 u}-1) & , u \leqslant 1 / 2 \\ \beta(1-\sqrt{2(1-u)}), & u \geqslant 1 / 2\end{cases}
$$

To get triangle random variables $z$, some appropriate $\beta$ is chosen. On computers very reliable random number generators uniform on $(0,1)$ are available. On Honeywell $66 / 20$ we used FLAT to get a number $U \sim$ uniform $(0,1)$ in each step. We check if $U$ is greater or less than half and accordingly compute $z$ from equation (Al). A Fortran program for the process follows. 


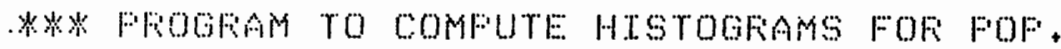

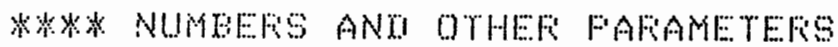

DOUBLE FEECTETON $N(2,1), C, N X(2,1), \times(2,2), T F(2), F(2,2)$

INTEGER $6(000)$, WA $(100), W B(100)$, WC $(100), W$ W $(100)$

IMFITCIT DOUBLE FFECTSTON (A-F, ONT,X-Z)

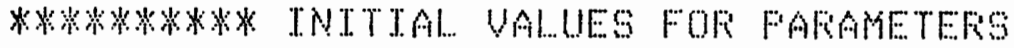

1008 M 91.1 .2

$T=(1)=0.7$

$T F(2)=-3$

$F x=\cdots, 1$

$F(1.4)=.89$

$F(1,9)=1$.

$F(2,1)=: 4$

$F(2,2)=0$.

For N $(1,1)+N(1.2)$

100

10 90

50

I. $=1.92$

AY $\cdots 0$.

$J=1,2$

BYY $=0$.

$C Y=0$.

IIYY $Y=0$.

VAY $=0$.

UBY $=0$.

VCY $=0$.

UIIY $=0$.

100 $3 x=1.500$

$3 \quad 0(\mathrm{~T})=0$

100 7 I $=1,100$

$W A(1)=0$

$W B(I)=0$

UC $(I)=0$

$W W(I)=0$

7 CONTTNUE:

FOF $N(1,1)+N(2,1)$

$50 \quad 50$

1.0 50

$I=1.2$

$X(T, U)=F(T, y)$

50

$1=1,2$

$A=0.000000333557711$

110 $3 \quad 1=1,900$

$30(\mathrm{I})=0$

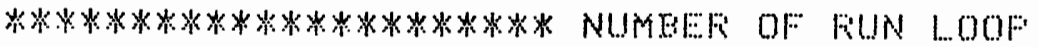

SPY $=0$.

AUL...M

SQL... $M=0$

110 $5=19500$

TH $1=500$

$P=0.5$

K $=0$

$1 . .=0$

$N(1,1)=10$

$N(2,1)=1.0$.

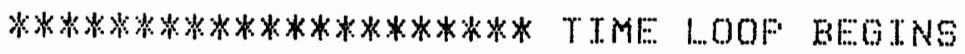

30

Kーバ+1. 
**** TFTANGULAR TITSTERUTHON: 6 LINES

$Y=F$ I...AT $(A)$

$I F(Y \cdot L T \cdot 0.5)$ Goro 71

$Z=1, \cdots(2+*(1, \cdots Y)) * * 0,5$

6oro 72

$71 . Z=(2 * Y) * * 0.5-1$.

********* FX TS MEASUFE OF FLUCTUATTON: BETA

$72 \times(2,1)=0.4+F \times * z$

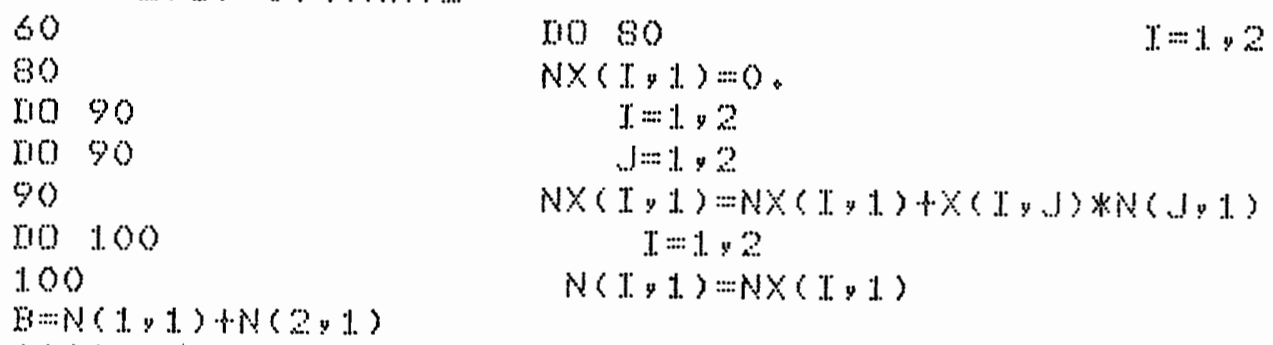

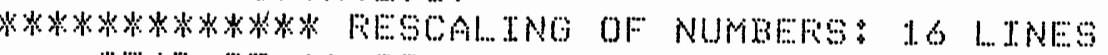

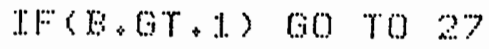

TF(K゙, GT + IH) OO TO 23

I.H:

$100 \quad 19 \quad \mathrm{~T}=10$ y 500

$190(T)=0(T)+1$

23 CONTTNUE:

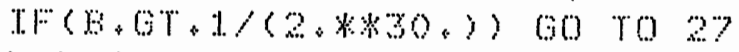

L... $=:$ L.........

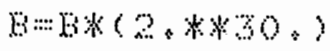

1.0 $1.7 \quad \mathrm{~T} \cdots 1.2$

$17 N(T, 1)=N(T, 1) *(2, * * 30$.

27 CONT TNUE:

TF $(B, 1, T \cdot(2, * * 30))$,00 T0 130

$1 . . .=1 . .+1.1$.

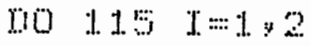

1. $1.5 N(T, 1)=N(I, 1) /(2+* 330$.

$\mathrm{B}=\mathrm{B}, \mathrm{B}, 2 . * 230$.

1.30

CONTINUE:

TF $(K \cdot L T+500) 60$ ro 30

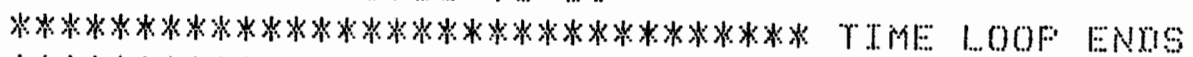

*************** CALCULATRON OF Y(T),U(T) AND

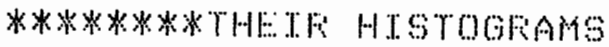

$F Y=N(1,1) / E$

$A Y=A Y+P Y$

VAY UAYHFYस*2

JY:FY 100.

IF $(J Y+1 . T 100)$ (30T0 31

WA $((100)=W A(100)+1$

Goro 32

31. WA $(J Y+1)=W A(J Y+1)+1$

$32 \quad F Y=N(2,1) / B$

$B Y=B Y+F Y$

UEY $=U E Y+F Y * * 2$

$K Y=F Y * 100$

IF $(K K Y+L Y+100)$ 00T0 33

$W E(100)=W E(100)+1$

coro 34 
$33 W B(K Y+1)=W E(K Y+1)+1$

3 . $F Y=N(1.91) / N(2$ y. 1$)$

CYY $=$ CY Y P FYY

VCY VCY TFY**2

$J Y=F Y * 1.0$

FYoN(2, y 1$) / N(1,1)$

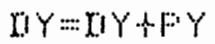

USY $=U Y Y+F Y * X_{2}$

KKY $=: F Y * 1.00$

IF $(K Y+1 . T+100)$ G0T0 37

WW $(100)=W(100)+1$

ooto 42

37 WD(KY+1) $=W[(1)+1)+1$

42 CONTTNUE:

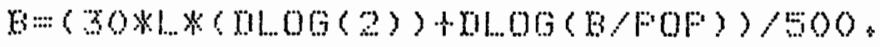

SM

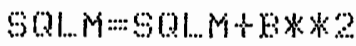

5 contrivuE

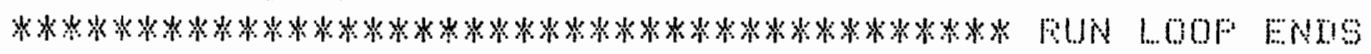

* * * * CAL CULATTON OF AVEFAGES ANO VARTANCES OF

*2* * FAFAMTEFS AND FRTN STATEMENTS

YYA=AY/WOO.

YYB: $=\mathrm{BY} / \mathrm{SOO}$.

DOA $\mathrm{CY} / \mathrm{SOO}$

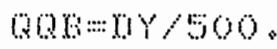

UYA $=(V A Y$ AY $W 2 / 500) /$.

UYB- (UEY-BYस*2/500.)/499.

VUA $=($ VCY C.CY*W2/500.)/49\%。

WUB $=$ (WDY DYW

AVL...M $=S M / 500$.

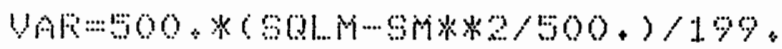

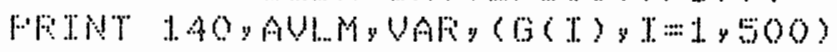

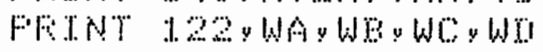

122 FOFMAT( $1 / 5(5 \times 20 T 3 \%)$

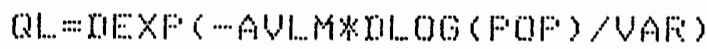

FETNT $190 \%$ QI...

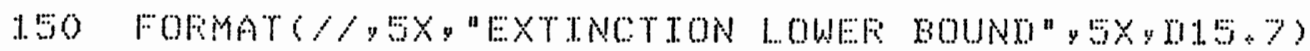

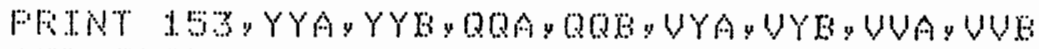

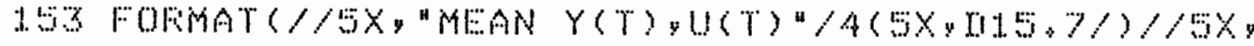

"UAFTANCE $Y(T)$, U(T)" $/ 4(5 X, 015,7 /)$ )

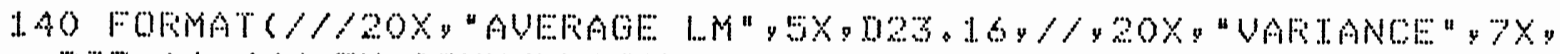

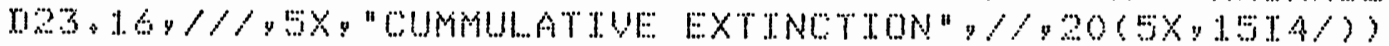

8 CONTTNUE:

STOF

ENoII 
APPENDIX B

ALOGRITHM FOR STATIONARY DISTRIBUTION AND ITS FORTRAN IMPLEMENTATION

Let $U$ and $p$ be bounded as indicated: $x_{1} \leqslant U \leqslant x_{2}$ and $p_{1} \leqslant p \leqslant p_{2}$. The intervals $\left(x_{1}, x_{2}\right)$ and $\left(p_{1}, p_{2}\right)$ are divided into convenient intervals:

$\varepsilon=\left(x_{2}-x_{1}\right) / N ; N$ intervals $I_{\alpha}, \alpha=1,2, \ldots, N$ with $\mu\left(I_{\alpha}\right)=\operatorname{Prob}\left\{u \in I_{\alpha}\right\}$

and $\quad \eta=\left(P_{2}-P_{1}\right) / M ; M$ intervals $S_{i}, i=1,2, \ldots, N$ with $p_{i}$ as midpoints of $S_{i}$

Now for each $p_{i}$ we calculate

$$
\begin{aligned}
& z_{1}=f\left(P_{i}, x_{1}+(\alpha-1) \varepsilon\right) \\
& z_{2}=f\left(P_{i}, x_{1}+\alpha \varepsilon\right.
\end{aligned}
$$

the maximum and minimum value of $U_{1}$ for every $\alpha .\left[z_{1}, z_{2}\right]$ would map on several $I(\beta), \beta=1,2, \ldots N$. calculate $c_{\alpha \beta}$ = fraction of $\left[z_{1}, z_{2}\right]$ in $I(\beta)$. This gives $\subseteq(i)$ a $N \times N$ matrix for all values of $\alpha$ and $\beta$. Since we do this for every $p_{i}$, we get a set of $m$ matrices $\underline{C}(i)$

calculate

$$
E[c]=\sum_{i=1}^{M} \subseteq(i) \nu\left(s_{i}\right)
$$


48

where

$$
\nu\left(s_{i}\right)=\operatorname{Prob}\left\{p \in S_{i}\right\}, i=1,2, \ldots, M
$$

$E[c]$ is Markov since $C_{\alpha \beta}(i) \geqslant 0, \sum_{\beta} C_{\alpha \beta}(i)=1$

The probability measure for the mapping of $U_{1}$ on $U_{0}$ is

$$
\begin{aligned}
\mu^{(1)}\left(I_{\beta}\right) & =\sum_{i, \alpha} \mu\left(I_{\alpha}\right) C_{\alpha \beta}(i) \nu\left(S_{i}\right) \\
& =\mu^{\prime} E[C]
\end{aligned}
$$

The probability density vector for $u_{t}$ is

$$
\mu^{(t)}=\mu^{\prime} E[\subseteq]^{n} \propto \Pi^{\prime}
$$

where $\Pi^{\prime} E[\subseteq]=\Pi^{\prime}$, the left eigenvector of $E[\subseteq]$. A Fortran implementation of the alogrithm follows. 
*****************FFOGFA TO COMFUTE STATIONAFY IISTFIBUTTON IMFICIT IOULHLE FFECISJON (A-HyOHZ)

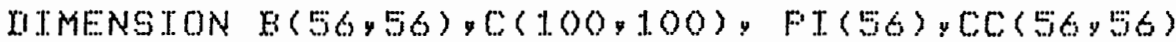

$$
\begin{aligned}
& \text { QM1 }=+85 \\
& \text { (RM2 }=1 . \\
& \times 1=0 . \\
& \times 2=1 . \\
& F 1=+3 \\
& F 2=+5
\end{aligned}
$$

************ INTEFUAL LENGTHS

$$
\begin{aligned}
& E I=(\times 2 \times 1) / 200 \\
& E 2=(F 2-F I) / 50 .
\end{aligned}
$$$$
\text { r.0 I. I. I.: } 1.960
$$

1.1. F I (T) $=1$.

[10 $12 \quad T=1.1 .00$

min $12, \mathrm{~J}=1.100$

I.2 $C(T, 1)=0$.

110 o $I=1.9100$

$F F=\cdots+(I+\cdots+\cdots) * 2$

100 4 $1=40,95$

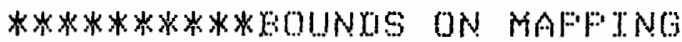

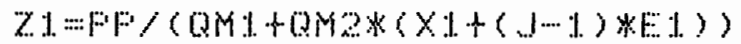

$Z 2=F=1(Q M 1+0 M 2 *(X 1+1+2) 1)$

$z 1=z 1.200$

$72=72 \times 200$

$A L=A B S(Z 2-Z 1)$

KI $=: T \mid I \times(Z)+1)+1$

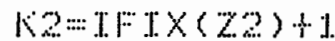

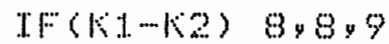

$80(3-39,(22-39)=(0)(1) 39$, $103-39)+1$.

$\operatorname{orro} 4$

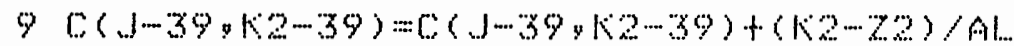

IF ( (KI-WK2)+LT.2) GOTO

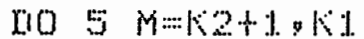

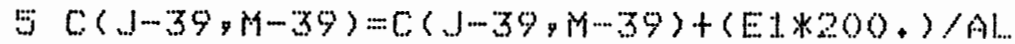

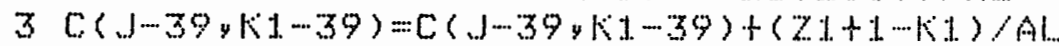

4 CONT TNUE

6 CONTINUE

********** PFINTING OF C $\{I, J\}$

IIO 15 I $I=1.90$

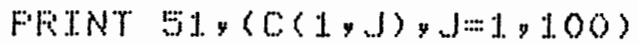

51 FOFMAT $(10(5 X, 10(F 4,2$. $2 X) /) /)$

1 CONTINUE

[10 $18 \quad T=1.96$

n.0 $18 \quad J=1,56$

1. $8 \operatorname{CO}(T, 1)=A B S(C(T, 1) / 50$,

$1033 \quad T=1.56$

FFITR $52,(\operatorname{CC}(T, 1)$, J $=1,56)$

52 FOFMAT $(5(5 X, 10(F A+2,2 X) /) / 1)$

33 CONTINUE

***********ITEFIATION FOF CC (I, J)

$M M=0$

$7 M M=M M+1$

II0 $2 \quad I=1,56$ 
$1102.5=1.56$

$2 B(T, y)=0$.

ก.10 $1.9 \quad \mathrm{~T}=1.56$

nio $19 k=1,56$

II0 $19 \quad,=1.956$

$19 B(\mathrm{~J}, \mathrm{~K})=\mathrm{B}(\mathrm{T}, \mathrm{K})+\mathrm{CC}(\mathrm{I}, \mathrm{J}) \times \mathrm{CC}(\mathrm{J} K)$

[10 $3 \%$ T. $=1.96$

$\operatorname{lin} 31 . \quad \mathrm{s}=1.60$

3. $\cos (x, y)=\cdots(\pi, y)$

$X X=C 0(1.10) / 0 C(1.11)$

$Y Y=C \mathrm{C}(2,10) / 0 \mathrm{C}(2,1.1)$

IF $(A B S(X X-Y Y)+1 . Y \cdot 10 * *(-5)) 00 T 021$

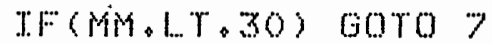

21. $5 \mathrm{~N}=0$ 。

w0 34 T. $=1.96$

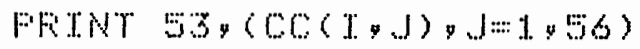

53 FONMAT(:) $2 x, 6(19.2 .2 X) /) / 1)$

34 CONTTNUE:

FFTNT 4\% y MiM

49 FOFMAT $3 X$, "FWER OF $2: " y, 3 X$, T 3 )

[10 $23 \quad 1=1.1 \% 6$

$23 \quad 5 N=3 N+C(1, .1)$

I10 22 I

22 F T (T) $=(00(1, \mathrm{~T}) / 8 \mathrm{~N}) * 1000$

FINT 60 OFT

50 FOFMAT (GX, "LEFT ETQEN UECTOF" $/ 56(6 \times .023 .16 /)$ ) STOF

ENTI 


\section{APPENDIX C}

A Fortran program to calculate birth sequences and relevant statistics is given beginning on the next page. 


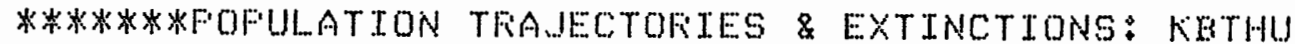
INTEGEF $\theta(50), H(5)$

IMWLICIT IOUBLE FRECISION (A-F,O-T,X-Z)

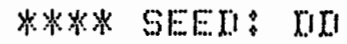

$\mathrm{CC}=42317765.77$

$011=3456721.001$

********* FEFTII..ITIES

$7 . z=0$

W10 1 MA $=1 .: 3$

IIXM $=0$

IIXX $\times 1: \cdots 10$

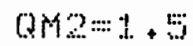

$0 M 3=2.20$

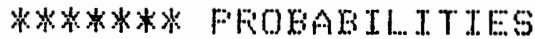

$F 1=0+5$

$F 2=0.20$

110 $3 \quad \mathrm{~T}=1.960$

$3(\mathrm{G}(\mathrm{T})=0$

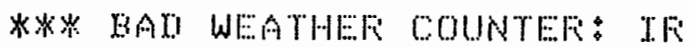

SM: $=0$.

AVI..M $=0$.

OCI $M=0$

$X M A X=0$

XMITN=1000.

XMAS $=\cdots 1$.

XMT. $S=100$

$z z=z z+02 z$

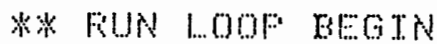

(10) $19: 1.200$

象*** COUNTERS

[10] 4 [ $[=1.95$

A $H($ I. $)=0$

$I R=0$

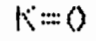

I... $=: 0$

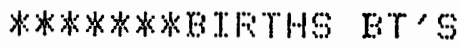

BT $3=1000$

$B T 2=1.096$

BT $1=1201$.

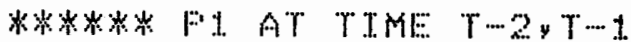

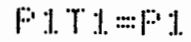

$\because 12=1 \% 1$

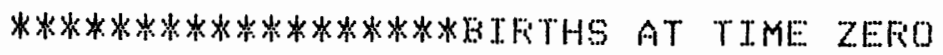

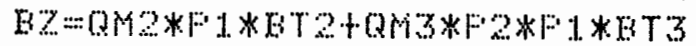

$\mathrm{BTX}=\mathrm{ET} 2$

BTY $T$ BT 1

$\mathrm{BT} Z=: \mathrm{BZ}$

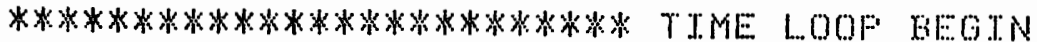

30

バ=ド+1.

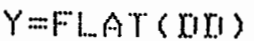

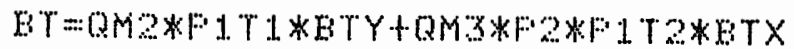

F. T2:-1T.1

$\operatorname{TF}(Y+\ldots=0.5)$ GOTO 51

$Z=\cdots F(\ldots A T(C)$ 
$F 1 . T 1=\cdots+\cdots z+z$

00003

Sil $Z=F I A T(C C)$

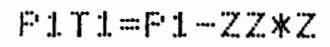

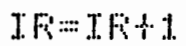

63 contrinuE:

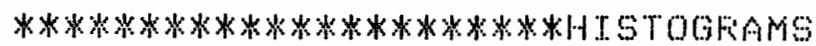

$\mathrm{IF}(\mathrm{l} . \mathrm{NE}+\mathrm{N})$ 0Oro 23

TF $(\mathrm{ET} \cdot 0 \mathrm{OT} \cdot 1000) 60 \mathrm{TO} 27$

$\operatorname{TF}(H(1)+E Q+1)$ OOTO 21

$H(1)=1$

$G(1)=6(1)+1$

2 CONTINUE:

TH

$\mathrm{IH}(H(2) \cdot E \mathrm{E}+\mathrm{H})$ GOrO 22

$\theta(2)=0(2)+1$

$H(2)=1$

22 CONTTNUE:

$T F(\mathrm{BT}+0 \mathrm{OT} \cdot 300) 00 T 027$

T.

$0(3)=0(3)+1$

$H(3)=1$

2A CONTTNUE:

IF(ET.0T.100) $60 T 027$

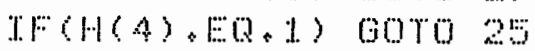

$\theta(A)=: G(4)+1$

$H(A)=1$

25 CONTTNUE

TF(BT.GT.1O) 6OTO 27

IF $(H(H), E \mathrm{O}, 1)$ GOTO 23

$0(5)=0(5)+1$

$H(\mathrm{i})=\mathrm{I})$

23 GONTTRUE

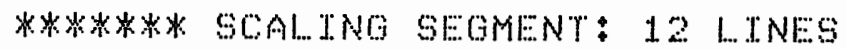

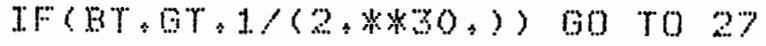

L...: $:=1 . . \cdots 1$.

$\mathrm{Br}=\mathrm{BT} *(2+* 230)$

BTY $=\mathrm{BTY} *(2, * * 30+)$

$\mathrm{BT} X=\mathrm{BT} \times\left(2, * * 30_{+}\right)$

B $Y Z=B T Z * 2 . * * 30$.

27 CONTTNUE:

JF(ET:LT. $(2 * * 30)) G$,0 TO 130

$L=: L+1$

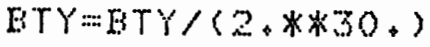

$\mathrm{BrX}=\mathrm{BTX} /(2, * 30$.

$\mathrm{BT}=\mathrm{BT} /\left(2+* \mathrm{x}_{30}\right)$

$\mathrm{Brz}=\mathrm{Brz} /(2+* 230)$

130

IF $F(K ゙+N E+10)$ GOTO 31

CONTTNUE:

$G(6)=K$

IIO $4 \% \quad T=1,5$

4.1. $\quad \mathrm{G}(6+\mathrm{S})=\mathrm{G}(\mathrm{G}+\mathrm{T})+\mathrm{H}(\mathrm{T})$

31. CONTTNUE:

IF $(K, N E+5 O)$ GOrO 32 
G( I. 2$)=\mathbb{K}$

IIO $42 \quad$ T: $=1$, 9

$42 G(12+I)=G(12+\mathrm{T})+H(I)$

32. CONTINUE:

IF (N゙.NE.10O) GOTO 33

$0(18)=: \mathbb{R}$

(10) $43 \quad 1=1,9$

$43(0(18+I)=0(18+I)+H(I)$

33 contrinue

II

$G(24)=5$

100 44 T.:-1. y

$440(24+\mathrm{T})=6(24+\mathrm{T})+H(\mathrm{~T})$

34 CONTINUE:

IF (K. NE. 300$)$ OOTO 35

$0(30)=k$

ए10 45 Tा: $=1.5$

$400(30+T)=0(30+T)+\cdots(I)$

$30 \mathrm{BTX}=\mathrm{BTY}$

BT $Y=\mathrm{H} Y \mathrm{Y} z$

$\mathrm{Er} Z=\mathrm{Br}$

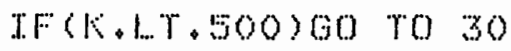

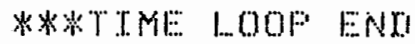

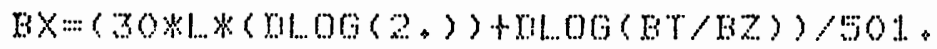

XMAS:MMAXI(XMAS \& BX)

XMTS:OMTNI (XMTS, EX)

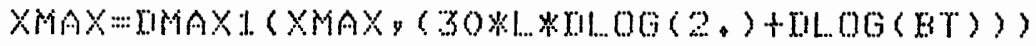

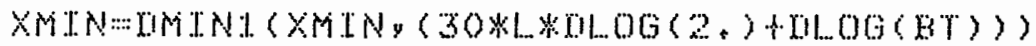

$S M=\sin \theta \mathrm{X}$

13 SOLM $601 \ldots M+B \times * 2$ ?

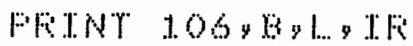

IOG FOFMAT (

5 CONTINUE

** RUN LOOF END

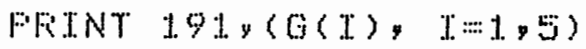

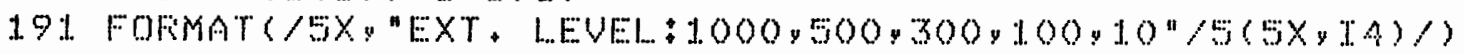

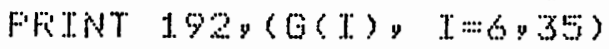

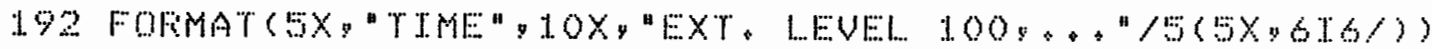

AVI..M $M$ SM 200 .

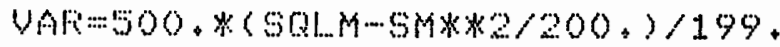

FIINT 1.40 Y AVI..M Y UAF

FFINT 122, WA Y WE , WG Y WW

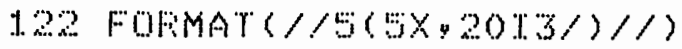

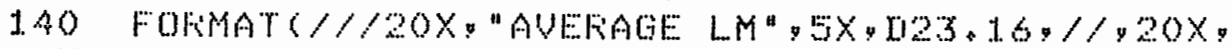

"UAFITANCE:" " "XX, [123,16/\%

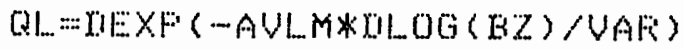

FRINT 1.:O Y QRL.

FFINT 153 Y YYA YYB , QRA , QRB, UYA YYE Y UUA Y UUE

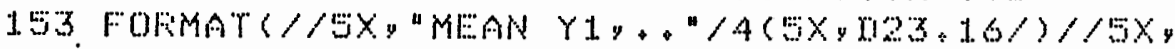

"VAFi UY1, " $/(B X, 123,16 /))$

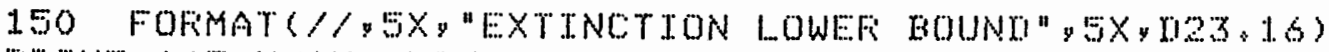

FATN 143 , XMAX, XMIN, XMAS y XMS

1. 43 FOFMAT $/ /$ EXX "N+LOO OF MAX\&MIN FOF \& LOO AU"

$4(5 \times,[123+16 /))$ 
1. CONTTNUE:

sTo:

ENO 